\title{
Nanoscale Drug Delivery and Hyperthermia: The Materials Design and Preclinical and Clinical Testing of Low Temperature-Sensitive Liposomes Used in Combination with Mild Hyperthermia in the Treatment of Local Cancer
}

\author{
Chelsea D. Landon ${ }^{1}$, Ji-Young Park ${ }^{2}$, David Needham ${ }^{3}$ and Mark W. Dewhirst*,1,2,3 \\ ${ }^{1}$ Department of Pathology, ${ }^{2}$ Department of Radiation Oncology, ${ }^{3}$ Department of Biomedical Engineering, Duke Univer- \\ sity, Durham, NC 27710, USA
}

\begin{abstract}
The overall objective of liposomal drug delivery is to selectively target drug delivery to diseased tissue, while minimizing drug delivery to critical normal tissues. The purpose of this review is to provide an overview of temperaturesensitive liposomes in general and the Low Temperature-Sensitive Liposome (LTSL) in particular. We give a brief description of the material design of LTSL and highlight the likely mechanism behind temperature-triggered drug release. A complete review of the progress and results of the latest preclinical and clinical studies that demonstrate enhanced drug delivery with the combined treatment of hyperthermia and liposomes is provided as well as a clinical perspective on cancers that would benefit from hyperthermia as an adjuvant treatment for temperature-triggered chemotherapeutics. This review discusses the ideas, goals, and processes behind temperature-sensitive liposome development in the laboratory to the current use in preclinical and clinical settings.
\end{abstract}

Keywords: Low Temperature-Sensitive Liposomes, hyperthermia, drug delivery, cancer.

\section{INTRODUCTION}

The purpose of this review is to provide an overview of temperature-sensitive liposomes in general and the Low Temperature-Sensitive Liposome (LTSL) in particular (the commercial name of the doxorubicin-loaded version is ThermoDox ${ }^{\circledR}$ ). This LTSL was designed to release drug rapidly upon a temperature trigger using mild hyperthermia at $41-42^{\circ} \mathrm{C}$. Its basic design to load and retain drug while evading the body's defenses is based on a wealth of information about liposomes that was gained over a period of 40 years. These studies include basic research and preclinical and clinical investigations that have led to the most advanced nanoscale drug delivery system in clinical therapy. Several recent reviews have detailed much of this work. In 2006, Immordino et al. [1] wrote a comprehensive review of the extensive literature on conventional liposomes and in particular focused on stealth technology, summarizing preclinical and clinical data relating to these principal liposome formulations. It also discusses the emerging trends at the time of this promising technology. Two years prior to this review, Allen and Cullis [2] provided an insightful account entitled, "Drug delivery systems: entering the mainstream". Torchillin [3] also discussed advances concerning liposomes as pharmaceutical carriers, identifying gene delivery and cancer therapy as the principal areas of interest; however this review recognized that while new trends must be identified and exploited that there are limitations with conventional liposome technology. More recently in 2008, Jesorka and

*Address correspondence to this author at the Box 3455, Duke University Medical Center, 201 MSRB I, Durham, NC 27710, USA; Tel: 919-6844180; Fax: 919-684-8718; E-mail: dewhi001@mc.duke.edu
Orwar [4] focused on technologies and analytical applications of liposomes as analytes and as functional components in a variety of new analytical systems, such as liposome immunoassays.While temperature-sensitive liposomes have been studied since the late 1970's, our LTSL first reported in 1999 has gained prominence in the last 5-6 years but was absent from these earlier reviews. We did provide a book chapter in 2006 [5] that compared and contrasted conventional and stealth liposomes with the new LTSL concept. Recently, Koning et al. [6] reviewed how hyperthermia can influence liposomal drug delivery by comparing and contrasting the pros and cons of extravasation from tumor vasculature and how hyperthermia can increase vascular permeability resulting in increased levels of liposome accumulation within the tumor tissue, including interstitial drug release and intravascular release of drug (which does not require liposome extravasation). Together these recent reviews cover most of the preceding literature on liposomes and their myriad applications. As the initiators and developers of this new low temperature formulation, in this review, we: 1) give a more materials design account of the LTSL and highlight the likely mechanism behind temperature-triggered drug release from the LTSL (depicted in Fig. 1), 2) focus on results from preclinical and clinical testing, 3) discuss the technical challenges of this approach, which involves devices that apply hyperthermia to reach the desired temperature range for optimal drug performance and 4) discuss the types of cancer that can benefit from this form of therapy.

\section{The Drug Delivery Problem}

Two of the greatest hurdles towards achieving cures with traditional chemotherapeutics are systemic toxicity and bioavailability at the tumor site (i.e., free drug is toxic to 


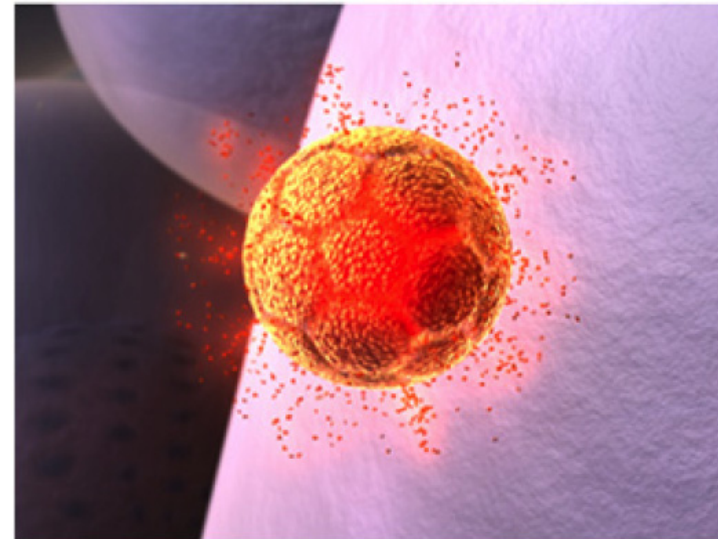

Fig. (1). Schematic of temperature-triggered drug releasing liposome (with kind permission from Celsion Corporation).

normal cells and achieves peak plasma concentrations at only 5 minutes post-bolus injection [7]). In order to enhance the biodistribution of these drugs, reduce free drug toxicity, and favor tumor accumulation [8,9], drug delivery research, preclinical testing, clinical evaluation, and commercial development [10] have principally focused on phospholipidbased liposomes $[11,12]$. Other self-assembling systemspolymeric micelles formed from amphiphilic block copolymers [13-16], polymer surfactant polymersomes [17], and covalent-linked structures, such as polymer-drug conjugates [18-21] and dendrimers [22, 23] —are still in research and development stages. Beginning in the 1970's, initial attempts to encapsulate drugs inside liposomes managed to successfully overcome toxicity issues of free drug [10], albeit at the expense of reduced drug availability for the encapsulated version. Furthermore, these traditional phospholipid liposomes were rapidly cleared by the reticuloendothelial system (RES) [24-26], limiting bioavailability. A major breakthrough in tumor drug delivery of drug-laden liposomes came when circulation times were extended due to the employment of PEG-lipid (or "Stealth") technology [27] or the maximal increase of membrane compressibility moduli [28] using saturated lipids (e.g. sphingomyelin and cholesterol) [29]. These strategies maintained the reduced toxicity of earlier liposome encapsulants, (by avoiding opsonization [30, 31]), extended the liposomes' circulation half-life from minutes to days, and exploited the hyperpermeability of tumor vasculature to achieve selective penetration [32-34]. Interestingly, it was shown in 1999 that the inclusion of PEG-lipid in a very low-compliant DSPC: cholesterol (3:2 molar ratio) membrane had little enhancing effect over actual drug delivered to animal tumors [35], demonstrating that a non-PEGylated high-modulus DSPC/ cholesterol bilayer remains as un-opsonized as one coated with PEG.

In vivo studies found that liposomes and other nanoparticles could achieve tumor-specific perivascular accumulation simply through passive extravasation [2, 36, 37]. However, the distribution pattern was found to be extremely heterogeneous and susceptible to large variations in vascular permeability $[38,39]$. With the totality of data demonstrating vascular permeability being exclusively derived from animal models, there remains a lack of evidence that human tumor vasculature is in fact permeable to many current "nanoparti- cle delivery systems" [40]. Even if the tumor vasculature is permeable to a $100 \mathrm{~nm}$ liposome, the relatively large size of the nanoparticle limits its penetration depth to one or two cell layers from blood vessels [41]. Furthermore, drug release rates (leakage) are usually so low that tumor cells might not even be exposed to drug concentrations high enough to achieve cell death [42-44]. Consequently, decades after the introduction of liposomal chemotherapeutics, delivering the drug at therapeutic and bioavailable concentrations to all tumor cells has not been achieved by conventional designs, necessitating other novel approaches towards encapsulated-drug delivery.

Temperature-sensitive liposomes represent just such an approach and have proven to be the most promising new technology to enter the field. Specifically, LTSL, which initiates local drug release using mild hyperthermia, has shown dramatic results in preclinical studies and is currently undergoing phase III clinical trials. These results are achieved most likely through intravascular release of the small molecule drug and its subsequent diffusion into the tumor mass, thereby avoiding reliance on the extravasation of the much larger carrier $[45,46]$. It is through this aspect of hyperthermic release that LTSL, in combination with radiofrequency (RF) thermal ablation and traditional microwave waveguide applicators, is now making a significant clinical impact on local drug delivery in several tumors types, including hepato-cellular carcinoma and chest wall recurrences of breast cancer [47-49].

\section{Regional Hyperthermia and its Combination with Liposomes}

Hyperthermia has been used in the treatment of disease, specifically cancer, for many years, and as depicted in Fig. (2), and discussed in more detail in the section on preclinical and clinical studies, hyperthermia has many therapeutic benefits. In addition to its direct cytotoxicity, it has also been shown that many chemotherapeutic drugs (e.g. doxorubicin and cisplatin) have a synergistic interaction with hyperthermia, resulting in an enhanced cytotoxic effect [50].

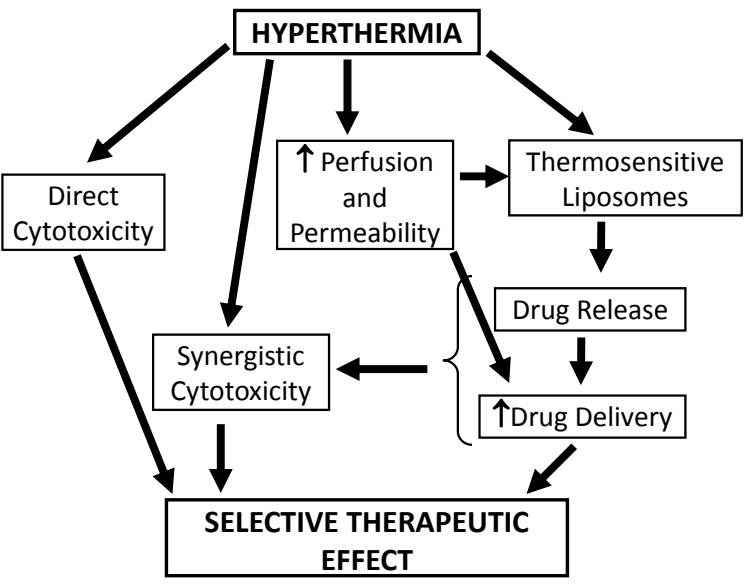

Fig. (2). Flow diagram depicting the multifactorial therapeutic benefits of hyperthermia. Hyperthermia enhances drug delivery and efficacy by increasing vascular perfusion and permeability and by enabling drug release from thermosensitive liposomes. Hyperthermia itself can be cytotoxic, but enhanced cytotoxicity is the result of the increased drug delivery and the synergistic interaction hyperthermia has with many anticancer drugs. 
Hyperthermia treatment is known to increase tumor blood flow and vascular permeability and may therefore increase drug delivery to the site of the tumor. Studies have also shown that hypoxic regions of the tumor typically do not accumulate high drug concentrations due to a relative lack of perfused microvasculature. Hyperthermia can be used to reduce these hypoxic regions by increasing blood flow, resulting in increased oxygen and drug delivery to these resistant regions. Regional hyperthermia is also a useful adjuvant treatment resulting in increased drug delivery and drug targeting, particularly in the case of liposomes. Both nontemperature-sensitive and temperature-sensitive liposomes benefit from the effects of regional hyperthermia. Hyperthermia increases liposome extravasation in animal models $[40,51]$ and, more importantly, in spontaneous tumors [52].

Traditional temperature-sensitive liposomes require relatively high temperatures $\left(42-45^{\circ} \mathrm{C}\right)$ to induce drug release [53-55]. Temperatures in this range are not readily achievable throughout a tumor volume in the clinic because of patient pain during heating and potential for normal tissue injury [56-58]. In contrast, low temperature-sensitive liposomes release their contents at $39-42^{\circ} \mathrm{C}$. This temperature range that is readily achievable for superficial and even deep-seated tumors [59-63] (see Table 3).

Hyperthermia also occurs at the margin of tumors treated with thermal ablative techniques. Although RF ablation effectively destroys the central tumor mass by raising the temperature to $\sim 65^{\circ} \mathrm{C}$, the temperature drops precipitously back to body temperature away from the heating locus, allowing microscopic deposits of tumor cells at the periphery to escape treatment [64]. Hyperthermia-enhanced liposomal formulations are hypothesized to be especially useful for combating these residual cells as temperatures that are below the thermal ablation threshold will still effectively release doxorubicin from circulating ThermoDox ${ }^{\circledR}[48,49]$ (Fig. 3).

With the introduction of the LTSL in 1999 [65], and demonstration of its in vivo efficacy in 2000 [66], thermal therapy coupled to temperature-triggered drug release has been reborn, as evidenced by the $30 \%$ of abstract presentations featuring this topic at the most recent ESHO meeting this past May 2010. While several new formulations are still in a preclinical setting [67-69], ThermoDox ${ }^{\circledR}$ (doxorubicin thermal-sensitive liposome being developed by Celsion Corporation) is being taken through human clinical trials and is setting the stage for formulation and testing of other temperature-sensitive chemotherapeutic drugs including cisplatin, as well as imaging agents that report on heatability, perfusion, and small molecule delivery [45].

\section{COMPOSITION, STRUCTURE, AND PROPERTIES OF LOW TEMPERATURE-SENSITIVE LIPOSOME DESIGN}

In this section, the review will address

2.1. Materials Design of Low Temperature-Sensitive Liposomes (LTSL)

2.2. Phase Transition Temperature $\left(\mathrm{T}_{\mathrm{m}}\right)$ of LTSL

2.3. Grain Boundary Structure of LTSL

2.4. LTSL Permeability

2.5. LTSL-Doxorubicin: Doxorubicin Loading and Release

2.6. Suggested Mechanism for Temperature-Triggered Drug Release from LTSL

2.7. Other Temperature-Sensitive Liposome Studies

2.8. LTSL Summary

\subsection{Materials Design of Low Temperature-Sensitive Liposomes (LTSL)}

In 1978, Yatvin et al. [54] developed the first temperature-sensitive liposome and reported that "in a test system in vitro, protein synthesis by Escherichia coli is inhibited and killing of the cells is enhanced by heating neomycin-

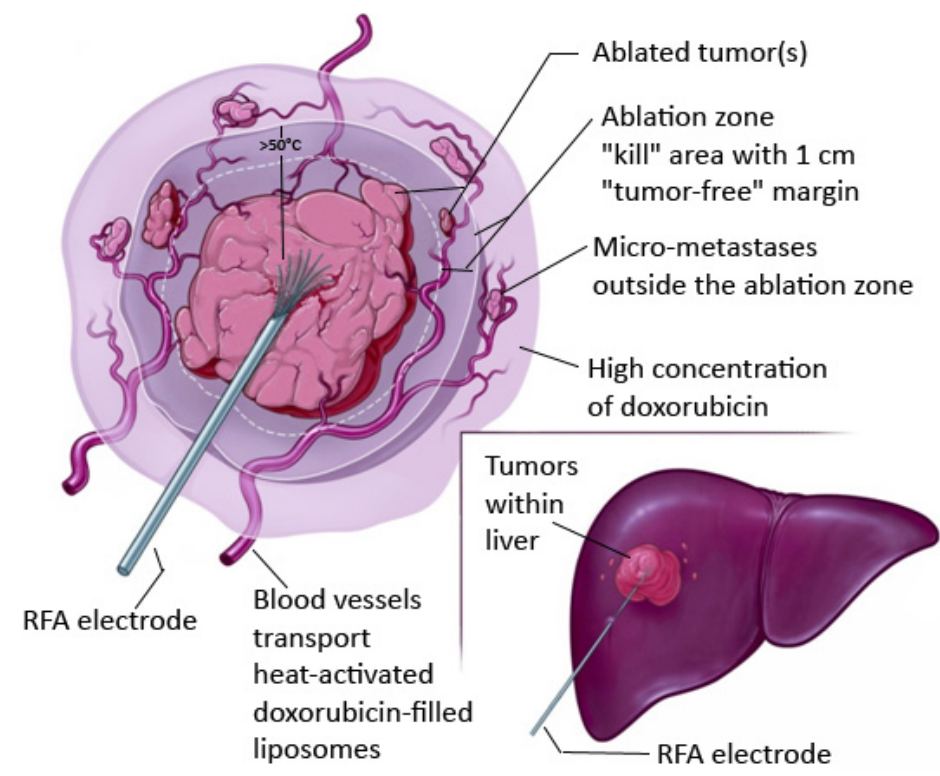

Fig. (3). Schematic showing how thermal ablation alone would miss the microscopic deposits of tumor cells around the tumor periphery, but how, with ThermoDox ${ }^{\circledR}$ in the blood stream, drug release is triggered in the $39-50^{\circ} \mathrm{C}$ thermal zone (with kind permission from Celsion Corporation). 
containing liposomes to their phase transition temperature to maximize drug release. In the presence of serum the ratio of release at $44^{\circ} \mathrm{C}$ to that at $37^{\circ} \mathrm{C}$ can be made greater than 100:1, suggesting possible applications in the treatment of tumors or local infection". The membrane lipid composition was based on DPPC (dipalmitoylphosphatidylcholine) which has a phase transition at $41.5^{\circ} \mathrm{C}$. The longer chain lipid DSPC (distearoylphosphatidylcholine) was also added in a 7:1 ratio of DPPC:DSPC. The addition of DSPC to the formulation raised the transition temperature of the ideal solid solution bilayer such that the liposome maximally released its encapsulated material in the temperature range of 43$45^{\circ} \mathrm{C}[53,54,70,71]$ - a range that is difficult to achieve clinically. Despite this drawback, Huang et al. [72] did demonstrate that doxorubicin-loaded temperature-sensitive liposomes showed increased therapeutic efficacy when combined with hyperthermia; however, this formulation was abandoned when it became clear that these "traditional thermal-sensitive liposomes" had a very short circulation halflife (this was pre-Stealth ${ }^{\circledR 1}$, i.e., pre-PEGylation of liposomes), formed aggregates, and released drug slowly ( $\sim 30$ minutes or more) [53]. Many of these issues were resolved in 1996 with the invention of the LTSL, a PEGylated temperature-sensitive liposome that rapidly releases contents in response to a heat stimulus within the mild, clinicallyachievable hyperthermia range of $40-42^{\circ} \mathrm{C}[46,66,73,74]$.

Preclinical investigation into the efficacy of LTSL has demonstrated its superior anti-tumor properties, as a result of its ability to deliver drug to the tumor at concentrations up to 30 times greater than those achievable with free drug and 3-5 times greater than those of traditional liposomes [46, 75]. Thus, LTSL represents a novel method by which to maintain the toxicity benefits of sequestering drug until it reaches the tumor, where mild hyperthermia can then act as a tumorlocalized release trigger ensuring that minimal drug exposure occurs in normal tissue. This LTSL design also overcomes the obstacles of vascular heterogeneity and limited penetration associated with the usual extravasation paradigm and, instead, effectively serves as a continuous intravascular infusion of drug originating at the tumor site. By this new mechanism, LTSL can facilitate the high intravascular drug concentrations that are necessary to drive cellular drug uptake and, most importantly, increase drug penetration further from vessels [76].

The main purpose of this new formulation is to achieve the conflicting goals of sufficient membrane stability for the encapsulation and retention of drug, a relatively long circulation half-life in the blood stream, and an ultrafast drug release when it enters the warmed tumor vasculature. This release rate needs to be faster than the transit time of the LTSL through the local tumor region in order to respond to applied hyperthermic temperatures. To achieve this, the known anomalous permeability that is associated with the main solid - liquid melting temperature of the acyl lipid chains was utilized [77-81]. With a phase transition temperature $\left(\mathrm{T}_{\mathrm{m}}\right)$ of $41.5^{\circ} \mathrm{C}$, DPPC was selected as the main component of the liposome membrane in order to set the main bilayer transition just above body temperature and in the mild hyper-

${ }^{1}$ Note, Stealth ${ }^{\circledR}$ was a particular commercial formulation that provided the optimum PEGylation to achieve long circulation drug delivery. thermia range. As with the temperature-sensitive formulation of Yatvin et al.'s and other's temperature-sensitive formulations, such saturated chain phospholipids show slow drug release. This drug release is slightly enhanced over nontransitioning bilayers [74], but is still too slow for therapeutic use, especially when liposome extravasation may be limited or non-existent. DPPC bilayers have a fairly low compliance and so are expected to be relatively (mechanically) stable in the blood stream. However, the grain boundary structures of the solid bilayer have been shown to bind proteins that could be responsible for opsonization [82]. Therefore, in order to achieve extended circulation times and to "hide" the grain boundaries, the design option here was to include a few mol\% of the lipid conjugated PEG $^{2000}$ (DSPE$\mathrm{PEG}^{2000}$ ). As we have shown earlier, $\sim 4-5 \mathrm{~mol} \%$ of $\mathrm{PEG}^{2000}$ lipid was sufficient to cover the bilayer of the so called "mushroom-brush" boundary [83]. In several studies, in the absence of lysolipid, DSPE-PEG ${ }^{2000}$ was not observed to significantly affect the bilayer permeability of the liposome to drug when composed of DPPC and DSPE-PEG ${ }^{2000}$ alone [84-86] (but see later regarding the paper by Li et al. [87] where DSPE-PEG ${ }^{2000}$ has a strong effect on bilayers composed of DPPC and DSPC for permeability of carboxyfluorescein). Furthermore, with acyl chains being only $2 \mathrm{C}$ longer than DPPC, DSPE-PEG ${ }^{2000}$ was found to raise the bilayer transition by merely a fraction of a degree [unpublished data]. It was experiments that measured the solution-bilayer exchange rates of simple lipids and surfactants that actually prompted the invention to include lysolipid in the bilayer [88]. Inclusion of $\sim 10 \mathrm{~mol} \%$ of the lysolipid MSPC produced significantly higher membrane permeability, with the highest permeability occurring at $\mathrm{T}_{\mathrm{m}}[86,88]$.

\subsection{Phase Transition Temperature $\left(T_{m}\right)$ of LTSL: Influ- ence of Membrane Composition and Bathing Solutions}

$T_{m}$ is the melting temperature of the acyl chains of the lipid and is measured by the gain in their configurational entropy. This gain is measured by Differential Scanning Calorimetry (DSC), monitoring the endothermic events (i.e., that absorb energy in the form of heat) when a sample of lipid is warmed through this acyl melting transition [89]. In the equilibrated solid (or so called, gel) phase, DPPC molecules are ordered and condensed in crystalline subgel $\left(\mathrm{L}_{\mathrm{c}}\right)$ phase [90] where the hydrocarbon chains are fully extended. In this region, the head groups of DPPC are relatively immobile at the water interface [91]. Upon heating, DPPC undergoes a 'subtransition' to the $\mathrm{L}_{\beta}$ phase and 'pretransiton' to the $\mathrm{P}_{\beta}$ rippled gel phase characterized by smaller enthalpy and gradual increase of the head group mobility of DPPC. As temperature is further increased, DPPC hydrocarbon chains melt as it transitions from the gel phase to the $\mathrm{L}_{\alpha}$ liquid crystalline phase. During the main transition, the orientation of the C-C single bonds change from trans to a state where gauche configurations are present [91]. This transition has an enthalpy of approximately $0.4 \mathrm{kcal} / \mathrm{mol}$ and an activation energy of $3 \mathrm{kcal} / \mathrm{mol}$ [92]. The $\mathrm{T}_{\mathrm{m}}$ of the phospholipid bilayer is influenced by the hydrocarbon chain length, the acyl chain saturation of the host lipid (e.g. DPPC), and the electrostatic properties of its head group. In general, increasing the chain length, or increasing the saturation of the chains, increases the transition temperature [92]. 
Table 1. Phase Transition Temperature of LTSLs in Various Media [Unpublished Data]

\begin{tabular}{|c|c|c|}
\hline Composition (mol \%) & Media & $\mathbf{T}_{\mathbf{m}}\left({ }^{\circ} \mathbf{C}\right)$ \\
\hline \hline \multirow{3}{*}{$\begin{array}{c}\text { DPPC:MSPC:DSPE- } \\
\text { PEG }^{2000}(86.5: 9.7: 3.8)\end{array}$} & HBS & 40.87 \\
\cline { 2 - 3 } & Human Plasma & 40.86 \\
\cline { 2 - 3 } & Human Blood & 41.17 \\
\cline { 2 - 3 } & DI water & 41.30 \\
\hline \multirow{4}{*}{$\begin{array}{c}\text { DPPC:MSPC:DSPE- } \\
\text { PEG }^{2000} \text { (85.0:9.8:5.2) }\end{array}$} & HBS & 41.10 \\
\cline { 2 - 3 } & Human Plasma & 40.96 \\
\cline { 2 - 3 } & RPMI Blood & 41.02 \\
\cline { 2 - 3 } & & 41.05 \\
\hline
\end{tabular}

The loading and release of drugs like doxorubicin into and from the LTSL has been studied and reported [74, 86]. All these measurements were carried out in HBS buffer. Since it could be the case that fats, proteins, and other small molecules present in blood plasma could partition into the bilayer and therefore change its transition temperature and breadth, we have now measured the transition data $\left(T_{m}\right.$ midpoint, onset and end temperatures) for LTSLs in plasma and whole blood. These data are important for ensuring, as much as possible from in vitro experimentation, what the transition temperature and their concomitant drug release will be in vivo in the animal or patient's blood stream. While $\mathrm{T}_{\mathrm{m}}$ sets the mid-point of the lipid bilayer transition, knowing the transition range (onset, peak and end) is important. As shown later in Fig. (7), drug release starts on the low temperature onset shoulder of the DSC Heat Flow curve. As shown in Table 1 [unpublished data], the $\mathrm{T}_{\mathrm{m}}$ of the "Standard" LTSL lipid composition DiPalmitoylPhosphatidylCholine (DPPC), MonoStearoyl-PhosphatidylCholine (MSPC), and DiStearoyl-PhosphatidylEthanolaminePolyEthyleneGlycol $^{2000}$ (DSPE-PEG ${ }^{2000}$ ) was measured in DI water, buff- ers, human plasma, or blood. The phase transition temperature of LTSLs are remarkably unaffected by the medium they are in, likely due to their solid bilayers with the graftedPEG making an effective barrier. As surprising as this seems, there appears to be no other component in blood that has a significant enthalpic transition coincident with DPPC. These data are especially important for planning in vivo experiments and therapy.

\subsection{Grain Boundary Structure of LTSL}

One of the most interesting and crucial design features of the LTSL is the presence of grain structure in the liposome membrane. As depicted in Fig. (4), lipid membranes and lipid monolayers in their solid phase are made up of grains with grain boundaries, and even for the $100 \mathrm{~nm}$ diameter LTSL, faceted grain structure is evident. Thus, in line with theory [79, 82], it has been established that drug release from LTSLs occurs via grain boundary permeabilization when it is heated into the region of its $\mathrm{T}_{\mathrm{m}}$, and that this anomalous permeabilization in the region of $T_{m}$ is dramatically enhanced by the presence in the bilayer of a few mol\% lysolipid [74].

Grain boundaries in lipid bilayers are planar defects due to the imperfect crystalline arrangement of the molecules in the grain boundary. This defect separates the regions displaying different molecular-crystalline orientation (grains). The structure is formed when the bilayer is cooled from its melted liquid region into the solid ordered gel phase. As the solidification temperature is approached, nucleates of solid domains are formed within the less ordered liquid crystalline membrane $[79,82]$. These solid domains continuously grow such that the phospholipids in the gel phase are oriented in a crystal lattice-like structure. When these domains meet in the final solidified membrane, domain growth stops and the grain boundaries are formed. As depicted in Fig. (4), these nano-scaled defects are present in microscopic monolayers of other solidified lipids like DSPC on gas microparticles [93] and are also very evident as a faceted structure even in the $100 \mathrm{~nm}$ diameter liposome [94]. These grain boundaries

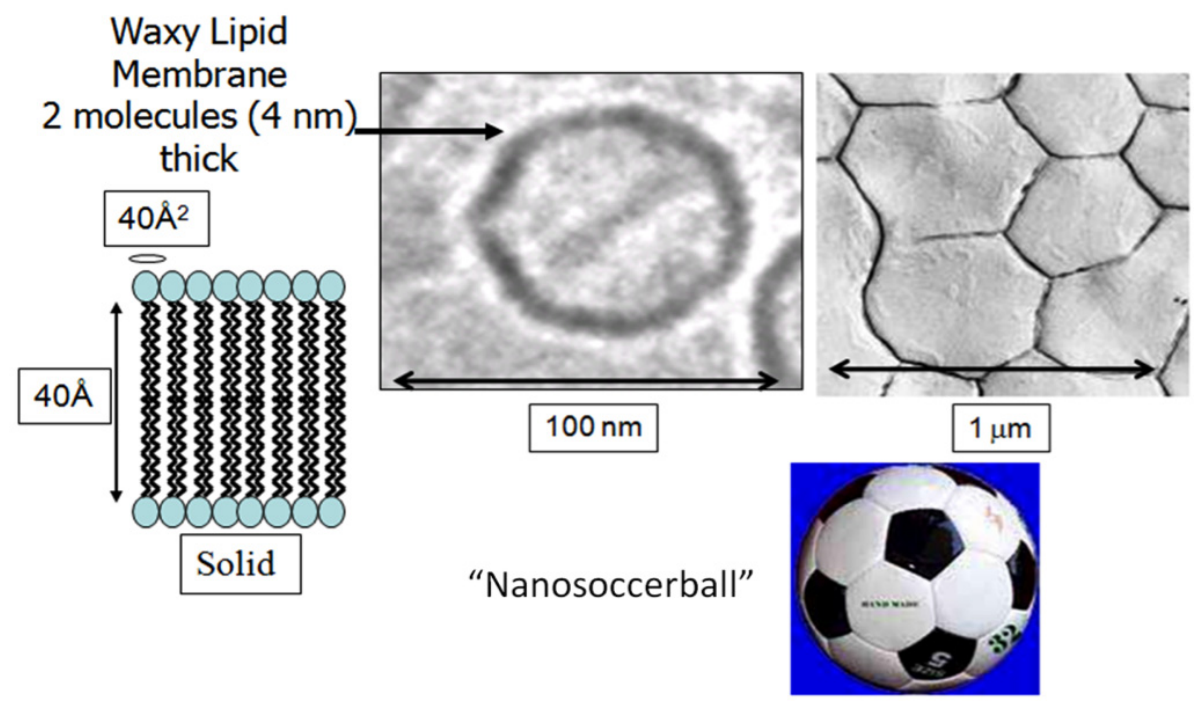

Fig. (4). Schematic showing (left to right) the solid all trans lipid bilayer that forms the faceted structure of the $100 \mathrm{~nm}$ Low Temperature-Sensitive Liposome [94]; grain structure is also evident in solid lipid monolayers on larger gas particles [93], prompting the term "Nanosoccerball". 
provide enhanced permeability of the membrane formed by saturated chain phospholipids, with the highest permeability occurring at $\mathrm{T}_{\mathrm{m}}$. This concept is essential for our LTSLs, since they are designed for ultrafast release. The saturated chain lysolipid MSPC was chosen because it is structurally and chemically compatible with the bilayer lipid DPPC. The incorporation of MSPC with DPPC achieves enhanced membrane permeability and also results in a slightly lower transition temperature as described in section 2.2. The DSC trace of liposomes composed of DPPC and MSPC is only slightly broadened compared to that of pure DPPC. This shows that the mobility of the bilayer is increased and that the bilayer could be slightly less stable than the bilayer of pure DPPC, a compromise that nevertheless still achieves effective encapsulation and stability in the blood stream.

\subsection{LTSL Permeability}

As described in sections 2.2. and 2.3. above, the LTSL formulation takes advantage of the anomalous permeability of lipid bilayer membranes at their transition temperature. This permeability occurs due to the characteristics of grain boundaries at $T_{m}$ and is enhanced by the inclusion in the bilayer of lysolipid. We characterized the ability of this lysolipid-based design to become permeable to small molecules, ions, and drugs (e.g. doxorubicin). Mills et al. [74, 95] evaluated the membrane permeability of LTSL using the ion $\mathrm{S}_{2} \mathrm{O}_{4}{ }^{-2}$ (dithionite) [96]. Using $1 \mathrm{~mol} \%$ of NBD lipids as the strongly absorbing component, they prepared two NBDlabeled liposomes (DPPC:MSPC(10\%):DSPE-PEG ${ }^{2000}(4 \%)$ and DPPC:DSPE-PEG $\left.{ }^{2000}(4 \%)\right)$ for permeability measurements. As dithionite was added at $30^{\circ} \mathrm{C}$ to NBD lipid-labeled liposomes (DPPC:MSPC(10\%):DSPE-PEG ${ }^{2000}(4 \%)$, NBDlipid, N-(7-nitrobenz-2-oxa-1,3-diazol-4-yl)-1,2-dihexadecanoyl-snglycero-3phosphoethanolamine, triethylammonium salt), the absorbance of NBD immediately decreased for the outer membrane because the outer membrane absorbance was quenched, but the inner membrane remained absorbent (Fig. 5). This experiment was repeated at increasing temperatures until dithionite ions permeated the bilayer. As shown in Fig. (5), the absorbance of the NBD-labeled lipids in the inner membrane of the liposomes only became quenched when dithionite had access to the liposome interior through the permeabilized membrane. The decrease in absorbance is faster in DPPC:MSPC $(10 \%)$ :DSPE-PEG ${ }^{2000}(4 \%)$ liposomes than for liposomes composed of DPPC:DSPE$\operatorname{PEG}^{2000}(4 \%)$ and peaks at $\sim 42^{\circ} \mathrm{C}$. These data demonstrate the extent to which lysolipid (MSPC) is responsible for the rapid permeability of the LTSL membrane at this phase transition compared to DPPC:DSPE-PEG ${ }^{2000}(4 \%)$.

\subsection{LTSL-Doxorubicin: Doxorubicin Loading and Release}

Following the demonstration of enhanced permeability by dithionite, the challenge then was to determine if and to what extent this permeability was retained for an actual drug. We chose to use doxorubicin because of the wealth of literature on doxorubicin loading, encapsulation, leakage, and its use in liposome-based delivery in other more traditional and PEG-liposome systems, including the commercial Stealth ${ }^{\circledR}$ liposome Doxil ${ }^{\mathbb{R}}[97,98]$.

Doxorubicin, a weak base cation with a pKa of 8.3 , is readily loaded by adapting the $\mathrm{pH}$ gradient loading (or remote loading) method [99]. For the LTSLs, we showed that this could occur at temperatures below the $\mathrm{T}_{\mathrm{m}}$ of the lipid (i.e., at $35^{\circ} \mathrm{C}$ ) and so developed the loading method for the LTSL-doxorubicin $[86,95]$. With regard to amount of drug released, Fig. (6) shows that the clinical formulation of LTSL-doxorubicin releases $80 \%$ of encapsulated drug within 20 seconds of being heated to its triggerable temperature of $41.3^{\circ} \mathrm{C}$ [86]. This is important to the in vivo mechanism since this rapid release time matches or is less than the transit time of liposomes through the tumor microvasculature, and LTSLs get ample time to release their drug in the heated tumor microvessels. Dewhirst et al. [100] demonstrated that median red cell velocity through tumor microvessels is approximately $0.54 \mathrm{~mm} / \mathrm{sec}$. The typical transit length through a tumor is many millimeters; therefore, the transit time will typically be far longer than 20 seconds, which provides sufficient time for drug release. Moreover, Chen et al. [101] used fluorescent red blood cells (RBC), and confirmed that

Fig. (5). Dithionite permeability of two membranes of liposomes composed of either (a) DPPC $(96 \%): \operatorname{DSPE}^{-P E G}{ }^{2000}(4 \%)$ or $($ b) DPPC(86\%):MSPC(10\%):DSPC-PEG ${ }^{2000}(4 \%)$ at $30,37,40,42$, and $43^{\circ} \mathrm{C}$. The absorbance of NBD slowly decreases in DPPC( $\left.96 \%\right)$ :DSPE$\operatorname{PEG}^{2000}(4 \%)$ liposomes (a) but quickly decreases in DPPC $(86 \%)$ :MSPC(10\%):DSPE-PEG ${ }^{2000}(4 \%)$ (b) due to increased bilayer permeability for liposomes composed of DPPC(86\%):MSPC(10\%):DSPE-PEG ${ }^{2000}(4 \%)$. Reproduced with permission from Mills [95]. 
the $\mathrm{RBC}$ velocity was $0.428 \pm 0.037 \mathrm{~mm} / \mathrm{sec}$ in untreated blood vessels but that after LTSL triggered doxorubicin release, the velocity decreased to $0.043 \pm 0.026,0.012 \pm 0.012$, and $0.003 \pm 0.003$, at 0,6 , and 24 hours, respectively, after the combined treatment of LTSL-doxorubicin and hyperthermia. These data show that while initial blood flow is already slow enough to allow for total release from liposomes that enter the heated tumor vasculature, upon drug release this blood flow is reduced even further, facilitating even greater drug release time.

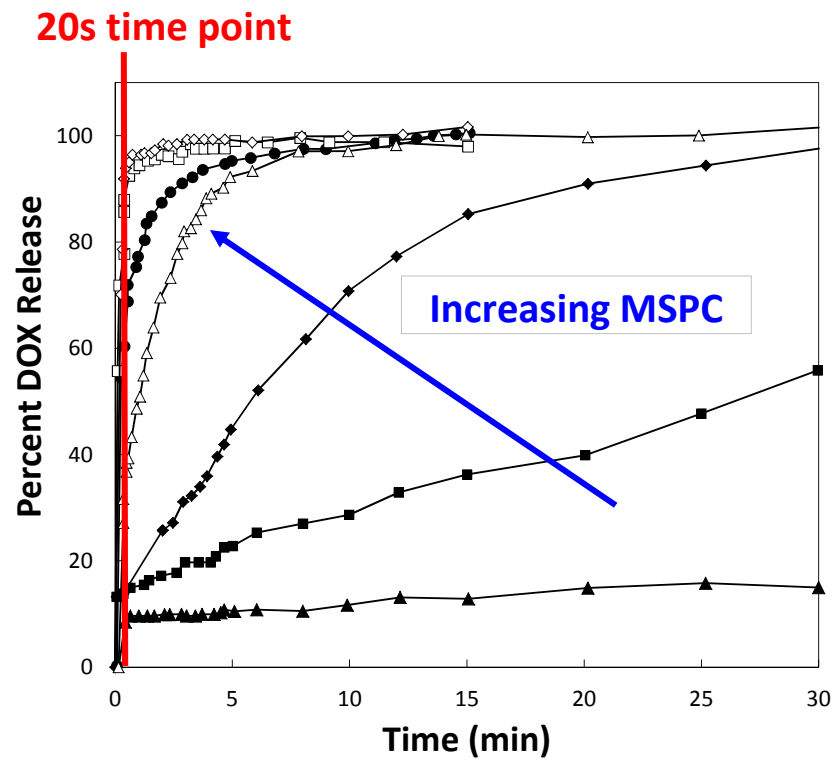

Fig. (6). Doxorubicin release vs. molar fraction of lysolipid in the bilayer (from $0 \mathrm{~mol} \%$ to $15 \mathrm{~mol} \%$ ) at $41.3^{\circ} \mathrm{C}$ [74]. (Bilayers also contained the usual $3.8 \mathrm{~mol} \%$ DSPE-PEG ${ }^{2000}$ ).

Fig. (7) shows the relationship between membrane permeability (measured as the release rate of doxorubicin) and the phase transition enthalpy and $\mathrm{T}_{\mathrm{m}}$ of the liposomes measured by DSC [74]. As can be seen, our basic starting formulation of DPPC:MSPC:DSPE-PEG ${ }^{2000}$ (86.5:9.7:3.8 $\left.\mathrm{mol} \%\right)$ has a transition temperature in de-ionized water of $41.3^{\circ} \mathrm{C}$, and actually starts to release drug on the low temperature shoulder of the excess heat flow curve, i.e., before any significant mass of solid phase lipid material has melted. Also, the maximum permeability rate coincides with the midpoint of the transition, where it is expected that the grain boundary area is also at its maximum [79]. Note also that the drug is retained at $37^{\circ} \mathrm{C}$ but releases maximally at $41.3^{\circ} \mathrm{C}$. Thus, the transition temperature of the LTSL and the peak in the content rate of release curve are coupled, i.e. drug release is also very sensitive to acyl chain composition of all lipids in the bilayer.

LTSL contains both MSPC ( 10 mol\%) and PEG-lipid ( $\sim \mathrm{mol} \%$ ), so we evaluated the role of each of these components separately (with DPPC). With MSPC at $10 \mathrm{~mol} \%$ and no PEG-lipid, the release of doxorubicin was slower, reaching 50\% releas in 6 minutes [86]. The addition of just 1.3 mol\% DSPE-PEG ${ }^{2000}$ restored the rapid release we had seen for the standard LTSL composition. When DSPE-PEG ${ }^{2000}$ was included in DPPC liposomes at concentrations from zero to $15 \mathrm{~mol} \%$, no enhancement of doxorubicin release was seen when carried out at the peak of each bilayer's transition temperature [86]. Similar results were found by Banno et al. [102] who investigated the roles of DSPE-PEG ${ }^{2000}$ and MSPC in the functional performance of doxorubicin-loaded LTSL. They showed that varying the PEG-lipid concentration $(0-5 \mathrm{~mol} \%)$ or bilayer orientation did not affect the release; however, as had been demonstrated previously (Fig. 6 and $[74,86])$, MSPC (0-10 mol\%) had a concentrationdependent effect on drug release. This indicates that lysolipid is essential for initially creating an enhanced permeability, but that a small amount of PEG-lipid is required to enhance it even further. The inclusion of PEG-lipid alone in a DPPC bilayer has no effect on permeability and in fact, slightly increases the transition temperature due to the presence of di-saturated C18 acyl chains. Interestingly, Li et al. [87] showed carboxyfluorescein release for a different lipid composition of DPPC/DSPC/DSPE-PEG ${ }^{2000}$ in a molar ratio of $80:(20-x): x(x=1,3,4,5,6,10)$ indicating a discrepancy that has yet to be resolved in the literature.

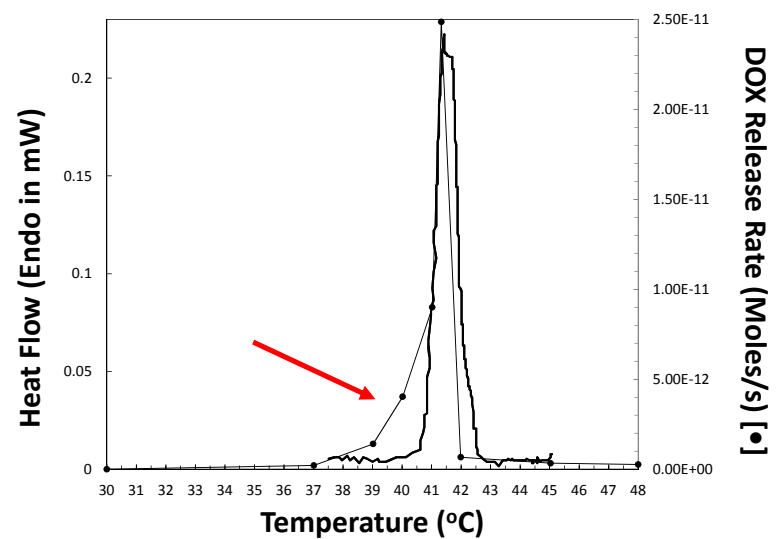

Fig. (7). Comparison between the Differential Scanning Calorimetry (DSC) thermal profile and the doxorubicin (DOX) release rate for LTSL-doxorubicin [74].

Thus, after evaluating membrane permeability to dithionite [74] and release of contents markers like carboxyfluorescein [86] and the drug doxorubicin [86], a final formulation was reached. The LTSL design is $100 \mathrm{~nm}$ in diameter and is comprised of DPPC (86.5 mol\%):MSPC (9.7 mol\%): DSPE-PEG ${ }^{2000}(3.8 \mathrm{~mol} \%)$. This formulation is patented [103, 104], and when loaded with doxorubicin is the commercial formulation now being tested in clinical trials [47-49] known as ThermoDox ${ }^{\circledR}$ [105]. Doxorubicin is loaded into LTSL by an already established method of remote, active, of $\mathrm{pH}$ gradient loading [99, 106-109]. The drug can therefore remain encapsulated until the membrane integrity is compromised by the thermal trigger where hydrogen ions can escape and the fiber bundles of doxorubicin can dissociate allowing the drug to be rapidly released in seconds from the liposome population.

\subsection{Suggested Mechanism for Temperature-Triggered Drug Release from LTSL}

We envision, as shown in Figs. (1 and 4), that the preexisting grain boundaries in the "Nanosoccerball" are the defects through which encapsulated drug can escape once the membrane integrity is compromised, but we are also interested in the mechanism at the molecular level. Several pieces 
of data including those presented above, together with theoretical considerations, lead us to the conclusion that drug release occurs at the transition through nanopores located at these grain boundaries formed by the presence of the lysolipid and enhanced (but not originated) by the presence of DSPE-PEG $^{2000}$.

To start the inquiry we consider the pure lipid membrane. As mentioned above, both Haest et al. in E.coli [77], Papahadjopoulos et al. in liposomes [81] and Marsh et al. in vesicles [78] demonstrated this anomalous permeability for lipid bilayers at their chain melting phase transitions. Also, as modeled later by Mouritsen et al. [79, 80], these grain boundaries are known to possess chain mismatch defects that result in permeability enhancement compared to the solid membrane below $T_{m}$, and the liquid membrane above $T_{m}$. We were curious as to exactly how anomalous this permeability might be for DPPC when compared to a liquid-lipid bilayer that did not possess a melting transition in the 41$42^{\circ} \mathrm{C}$ range. Thus, in a series of experiments by Mills and Needham [74], the permeability to dithionite ions at the transition temperature for DPPC was compared directly to that for Palimitoyloleoylphosphatidylcholine (POPC).

As shown in Fig. (8), the solid DPPC membranes begin to exhibit increased permeability to dithionite around $39^{\circ} \mathrm{C}$ and reach a modest peak at their phase transition midpoint due to melted and still-frozen chain mismatches at the grain boundaries, as predicted by Mouritsen $[79,80]$. With continued heating past $\mathrm{T}_{\mathrm{m}}$, there is a slight post- $\mathrm{T}_{\mathrm{m}}$ drop, but then the membrane permeability increases further and actually rises higher than the $T_{m}$ permeability value in what is now a purely melted $\mathrm{L}_{\alpha}$ phase bilayer with no grain defects, i.e., this is the high temperature permeability for lipid bilayers and has nothing to do with the transition anomaly. This is shown when we measure the same dithionite ion permeability for POPC liposomes that are in the liquid $\mathrm{L}_{\alpha}$ phase throughout the whole temperature range and are as close in composition to DPPC as possible (one saturated $\mathrm{C} 16$ chain and one unsaturated $\mathrm{C}=\mathrm{C}$ (at the 9 position) $\mathrm{C} 18$ chain). The permeability for this POPC liposome is higher than the solid phase DPPC, and is identical in the liquid $\mathrm{L}_{\alpha}$ phase for DPPC. At the transition, we see that the anomalous permeability of DPPC due to the grain boundary defects is only slightly higher than for a normal liquid membrane. We know from the work of Evans and Kwok [110], who used a convolution approach coupled with the "Clausius-Clapeyron" equation for the membrane surface to derive the thermal properties of the transition from the elastic compliance vs. temperature data, that at the phase transition of PC bilayers, both theory and micropipette experiment on giant lipid vesicles show a dramatic reduction in the area compressibility modulus which correlates with a decrease in membrane permeability. Also from Needham and Evans, [111], the bilayer thermal expansion coefficient is higher above the transition than below it, and causes the area per molecule to increase (lipid bilayer area density decreases). Since bilayer permeability increases with decreasing compressibility modulus and increasing molecular area, this explains the increasing permeability for the liquid phase bilayers of POPC and for DPPC above $T_{m}$. Given this behavior for the host lipid, we wanted to investigate the role of lysolipid, in so dramatically increasing this boundary-transition permeability as shown by the almost 10 -fold increase in membrane permeability with the inclusion of either the mono-C16 MPPC or the monoC18 MSPC lysolipid. Lysolipids do not form bilayers unless complexed in a 1:1 mole ratio with other molecules like cholesterol [112-115]. In aqueous solution they are monomeric solutes or associate into micelles above their critical micelle concentration (CMC). For MSPC, this is around $1 \mu \mathrm{M}$. Thus, when a lipid bilayer is exposed to a sub-CMC solution of lysolipid, the lysolipid enters the bilayer and can cross to the inner monolayer, as was demonstrated by Needham et al. [88]. If the bilayer is exposed to a more concentrated lysolipid solution, $(100 \mu \mathrm{M})$, lysolipid rapidly enters and saturates the bilayer and essentially dissolves it. Our invention of the LTSL is predicated on lysolipid already being in the bilayer at about $10 \mathrm{~mol} \%$ (equilibrated with about $1 \mu \mathrm{M}$ MSPC solution), and so the bilayer is stable when made. When the temperature of this DPPC:MSPC (90:10) solid solution bilayer is raised to $T_{m}$, the grain boundaries melt first, the chain mismatches occur, and then lysolipid is free to diffuse into this high energy region. From what was discussed above, three mechanism seem possible.

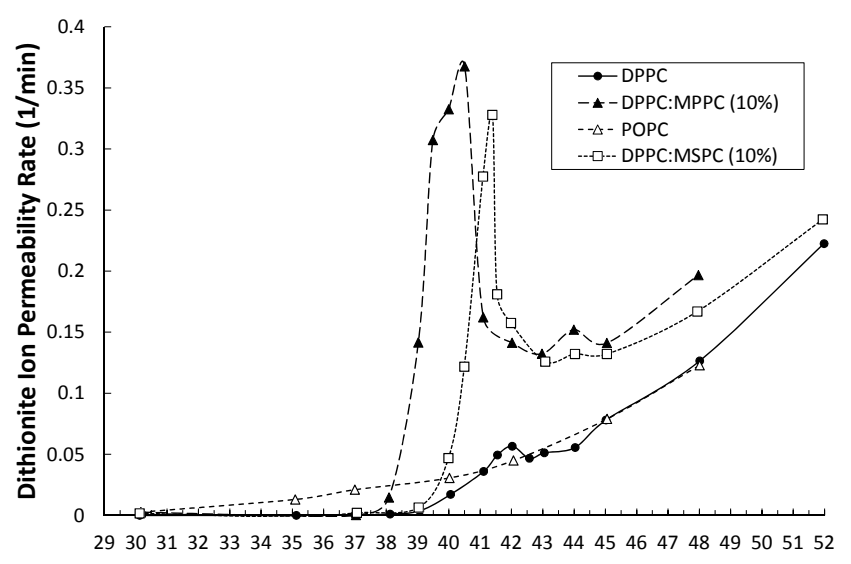

Temperature $\left({ }^{\circ} \mathrm{C}\right)$

Fig. (8). Dithionite ion permeability rates for DPPC, POPC and lysolipid-containing (MPPC and MSPC) membranes. All liposomes also contained $4 \mathrm{~mol} \%$ DSPE-PEG ${ }^{2000}$. At the phase transition temperature, permeability rates are $\sim 10$-fold higher for the lysolipidcontaining LTSL when compared to the pure DPPC bilayer [74].

The first would simply be that lysolipid reduces the membrane compressibility modulus at the $T_{m}$ region even more than occurs for single component membranes as shown by Evans and Kwok [110]. This could result in an even greater increase in membrane permeability. However, Zhelev has shown that the presence of lysolipid in the bilayer does not decrease the membrane compressibility [116], and so from this mechanical perspective a lysolipid containing bilayer has a very similar membrane compressibility to one without.

In the second, following the molecular exchange experiments of Needham et al. [88], which showed that lysolipid in solution would readily partition into the bilayer and could also be rapidly washed out of the outer monolayer, one of our original hypotheses was that lysolipid that was trapped in the solid phase below $T_{m}$ would leave the bilayer at $T_{m}$ thereby forming molecular scale defects through which 
drugs could escape. Similar conclusions were arrived at by Sandstrom et al., who [117] studied lysolipid membrane distribution and its retention in the LTSL composition. Dialysis experiments by Mills and Needham (in media that did not contain lipid or protein sinks for the lysolipid, just extensive dilution) showed that the lysolipid appears to sufficiently remain in the membranes to provide rapid drug release in the same temperature-controlled manner [74]. However, a more realistic evaluation of the in vivo scenario by Banno et al. [102] showed that lysolipid did dissociate from the circulating LTSL and that this might be mediated by the plasma protein and cellular membrane pools. While this may account for the slow destabilization of the LTSL in the blood stream and reduction in the drug to lipid ratio over a period of a few hours, it cannot be responsible for the rapid and triggered release seen for the heated sample.

We are therefore left with a third hypothesis that the tendency for lysolipid to form highly curved micelles results in it lining nanoporous defects in the lipid bilayer, probably at the grain boundaries. As summarized in the transverse bilayer schematics (Fig. 9), the level of membrane permeability at solid-liquid interfacial grain boundary regions is strongly dependent on the lipid membrane composition.

Fig. (9A), DPPC Bilayer in Phase Transition Region depicts the expected situation modeled by Mouritsen et al. $[79,80]$ where the chain mismatches between a solid mostly trans lipid in the solid phase do not line up exactly with the more liquid-like chains of the melted or melting lipid, and this creates a higher anomalous permeability. This is the situation for pure DPPC; as shown in Fig. (8), the membrane permeability to dithionite for DPPC Bilayers in the phase transition region is only marginally higher than a single component liquid phase membrane (palmitoyloleoylphosphatidylcholine) at the same temperature. This is also the level and mechanism of permeability observed by Haest et al. [77] and Papahadjopoulos et al. [81] for hydrated ions.

Fig. (9B), DPPC:MSPC Bilayer in Phase Transition Region where there is No Pore, as discussed above, shows that the presence of the chain vacancy defect that occurs by virtue of lysolipid occupying a lipid position in the bilayer has little if any effect on membrane compliance and therefore is not expected to decrease the permeability, simply by virtue of it being in the bilayer. Thus, we would expect that the anomalous permeability associated with the grain boundary mismatches would be similar to pure DPPC.

However, Fig. (9C), DPPC:MSPC Bilayer in Phase Transition Region with Enhanced Permeability Through MSPC Pore, shows how as the transition temperature is approached and the boundaries begin to melt, lateral lipid transport is increased and could allow more lysolipid to assume its preferred curvature (i.e. as a convex micelle), relaxing the planar bilayer structure by forming lysolipid-lined nanopores. As discussed previously, DPPC:MSPC has an enhanced permeability, but it is not until we add at least 1.3 mol\% DSPE-PEG ${ }^{2000}$ that the enhancement is increased such that drug is released at $\mathrm{T}_{\mathrm{m}}$ in just a few seconds. Thus, the schematic for Fig. (9D), DPPC:MSPC:DSPE-PEG ${ }^{2000} B i$ layer in Phase Transition Region with Enhanced Permeability Through MSPC Pore Stabilized by DSPE-PEG ${ }^{2000}$ shows how lysolipid forms the pores and the PEG-lipid must also accumulate to some extent during the melting process in order to stabilize them and keep them open. For small drugs, this presence of PEG polymer is unlikely to retard the permeability through the water-filled pore since the actual occupied volume is only a few percent of the polymer's excluded volume.

Data from other labs suggest that PEG-lipid included in a DPPC-DSPC mixture also enhances the permeability [87]. DPPC/DSPC/DSPE-PEG ${ }^{2000}$ in molar ratio of 80:(20-x):x. (where $\mathrm{x}=1,3,4,5$ ) show $85 \%$ release of carboxyfluorescein in 10 minutes, so chain mismatch defects (in this case, melting ideally mixed regions of DPPC:DSPC) seem to be a nucleation site for enhanced release even if lysolipid is not present. The existence of pores is not clear in this example.

Circumstantial evidence exists for the presence of lysolipid-induced nanopores in liquid lipid bilayers. The work of Zhelev et al. [118, 119] shows that the presence of nanopores in liquid lipid bilayers can be induced by the adsorption of the influenza peptide. The swelling of unilamellar vesicles held on the end of a micropipette suggested that there were stable and solute-selective pores with average size on the order of $5 \mathrm{~nm}$, which were able to selectively transport glucose and small ions from the surrounding buffer, but not sucrose, from the vesicle interior. Also when exposed to extensive freeze-thaw cycles, Ickenstein et al. [94] observed increasing formation of what appeared to be planar discs of bilayer in EM samples, indicating the tendency for solid phase LTSL to eventually break up at the grain boundaries and form bilayer discs where their edges are stabilized by lysolipid and PEG-lipid (i.e. analogous to Fig. (9D) with the domains of bilayer separated and stabilized at their edges with PEG-lipid).

To test the hypothesis that lysolipid forms pores in the LTSL membrane, Wright [86] conducted a series of experiments measuring the permeability of the LTSL bilayers to fluorescent dextrans. The limit of permeability occurred for a $20,000 \mathrm{Da}$ dextran, which from scaling theory would have a radius of gyration (diameter) of $\sim 5 \mathrm{~nm}$. Significantly, this diameter is approximately the same as the thickness of the bilayer. This leaves us with a simple model where micellarlike nanoporous defects of lysolipid form at the chain mismatch grain boundaries in the melting bilayer, as depicted in Fig. (9).

Finally, comparing Figs. (5 and $\mathbf{8}$ ), there appears to be a discrepancy in membrane permeability at temperatures above $T_{m}$. This is a complex issue. As both sets of data show, membrane permeability to both doxorubicin and dithionite peak at the phase transition temperature. Beyond that it must be recognized that dithionite is a much smaller ion, and its read-out requires quenching or absorbance-shifts by binding to NBD on the internal surface of the $100 \mathrm{~nm}$ liposomes once it has transited the bilayer from the bathing solution. Doxorubicin on the other hand is encapsulated and is rapidly released at the transition. In fact, when the sample is heated beyond $T_{m}$, drug is rapidly lost, and the driving force for transport is reduced. Also, as suggested by Mouritsen $[79,80]$, grain defects could exist up to $5-10^{\circ} \mathrm{C}$ beyond $\mathrm{T}_{\mathrm{m}}$. Thus, enhanced permeability (for the much smaller dithionite) could still be occurring but the larger doxorubicin may be limited to just the $T_{m}$ region. Interestingly then, for dithionite (in Figs. 5 and 8), we do show an attenuation of the release rate for dithionite at temperatures above the tran- 

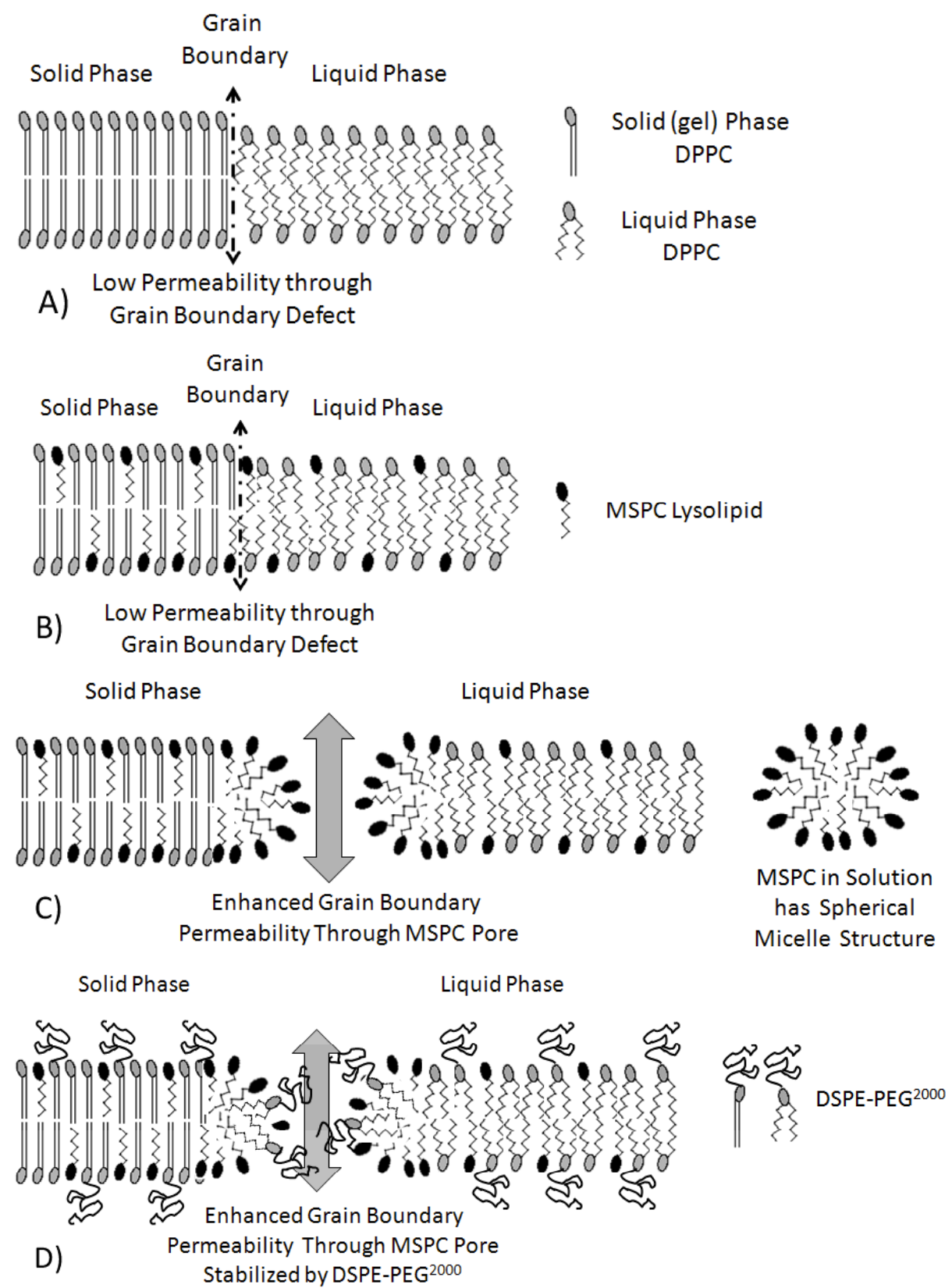

Fig. (9). Schematics of postulated defect structures that result in membrane permeability for: A) DPPC Bilayer in Phase Transition Region; B) DPPC:MSPC Bilayer in Phase Transition Region--No Pore; C) DPPC:MSPC Bilayer in Phase Transition Region with Enhanced Permeability Through MSPC Pore; D) DPPC:MSPC:DSPE-PEG ${ }^{2000}$ Bilayer in Phase Transition Region with Enhanced Permeability Through MSPC Pore Stabilized by DSPE-PEG ${ }^{2000}$.

sition temperature mid-point compared to the precipitous drop for doxorubicin, (Fig. 7). Furthermore, the permeability for dithionite slows to values that are consistent with even non-transitioning bilayers like POPC in this temperature range.

Note, as drawn to scale in Fig. (10), compared to doxorubicin, which is estimated from X-ray diffraction measurements on benzene to be $\sim 2.5 \mathrm{~nm}$ across [120], the dithionite ion (from its $\mathrm{C}_{2 \mathrm{~h}}$ trans structure in solution [121]) is only $0.65 \mathrm{~nm}$ in size.

The bottom line then seems to be that dithionite ion permeability is that for a much smaller ion and also requires a potentially contributory rate determining step of lipid head group signal quenching, while our measurements of the more clinically important doxorubicin release rates are maximal at $\mathrm{T}_{\mathrm{m}}$ and drop precipitously after that as the bilayer becomes more liquid and reseals to this larger drug molecule that cannot as rapidly go through the putatively remaining grain defects.

\subsection{Other Temperature -Sensitive Liposome Studies}

Motivated by the LTSL invention, modifications and potential improvements to the temperature-sensitive liposome formulation, including different lipid components are currently ongoing. For example, Lindner et al. [67] designed the temperature-sensitive liposomes composed of the 
novel lipid 1,2-dipalmitoyl-sn-glycero-3-phosphoglyceroglycerol (DPPGOG) and 1,2-dipalmitoyl-sn-glycero-3phosphocholine (DPPC). Hossann et al. [84] studied the influence of DPPGOG on in vitro stability of the liposome composed of DPPGOG, DSPE-PEG ${ }^{2000}$, and P-lyso-PC. They showed the release rate of the contents was significantly increased by incorporating DPPGOG or P-lyso-PC in their TSL formulations [84]. Also, Lindner et al. [122] formulated liposomes composed of HePC/DPPC/DSPC/ DPPGOG and showed that HePC increases the release rate of their TSL in a similar way to lysolipid in the presence of fetal calf serum. Interestingly, DPPGOG facilitates drug release from the liposome under mild hyperthermic conditions $\left(41-42^{\circ} \mathrm{C}\right)$ and leads to a substantially prolonged plasma half-life for the encapsulated drug [67]. Thus, in Lindner's formulations DSPE-PEG ${ }^{2000}$ is not required.

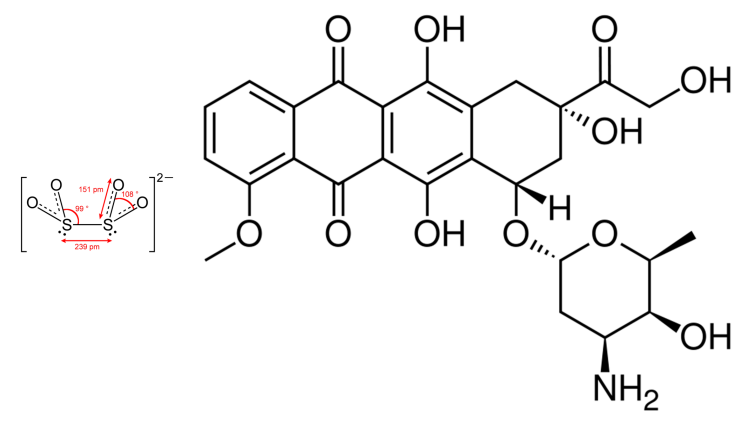

Fig. (10). Dithionite ion, $\sim 0.5 \mathrm{~nm}$ in size, compared to doxorubicin, $\sim 2.5 \mathrm{~nm}$ across; chemical structures and dimensions drawn to scale.

In other studies, temperature-sensitive liposomes have been designed using thermal-sensitive polymers. Hayashi et al. [123] studied temperature-sensitive liposomes composed of various phospholipids and coated with poly $(N$-isopropylacrylamide) that show a transition temperature near $32^{\circ} \mathrm{C}$. The copolymer modified liposomes show remarkable aggregation and fusion between $T_{m}$ and $T_{c}$, but much less aggregation occurs above the $T_{c}$. This study demonstrates that the copolymer chains coated on the surface of the liposome promote aggregation of the liposome due to temperature change, especially by the hydrophobic interaction between the copolymer chains and/or between the copolymer chains and the liposome membranes above $\mathrm{T}_{\mathrm{m}}$ of the copolymer [123].

Kono et al. [124] also used polymers and designed temperature-sensitive liposomes composed of dioleoylphosphatidylethanolamine modified with copolymers of $\mathrm{N}$ isopropylacrylamide and $\mathrm{N}$-acryloylpyrrolidine. These copolymer-modified liposomes were loaded with calcein to assess in vitro stability and release kinetics. They demonstrated that the inclusion of the anchor-bearing PEG derivative into the temperature-sensitive liposomes improves both temperature-sensitivity and content release kinetics.

Paasonen et al. [125] also reported on polymer coated liposomes with thermal-sensitive poly[ $N$-(2-hydroxypropyl) methacrylamide mono/dilactate] (pHPMA mono/dilactate) which has a $T_{m}$ at $42^{\circ} \mathrm{C}$. They studied temperature-triggered release from the polymer-coated liposomes. This liposome aggregated at approximately $43^{\circ} \mathrm{C}$ (above $\mathrm{T}_{\mathrm{m}}$ ) and showed a high degree of temperature triggered release above $T_{m}[125]$. Thus, several new studies have introduced the idea of modifications to the bilayer composition or surface of liposomes with temperature-sensitive polymers that retain temperaturetriggered release from the liposome. Such modifications may prove useful, but require further investigation.

\subsection{LTSL Summary}

In summary, the basic formulation starts with the three lipid components, DPPC, MSPC, and DSPE-PEG ${ }^{2000}$. Each component is necessary to achieve encapsulation and triggerable release of drug, and all three lipid bilayer components (and maybe even the drug) can influence the transition temperature of the composite bilayer, and therefore, the drug release kinetics and temperature range over which drug is released [74]. For example, compared to the host DPPC value, the introduction of di-C18 acyl chains of DSPE$\mathrm{PEG}^{2000}$ slightly increases $\mathrm{T}_{\mathrm{m}}$, and the mono-C18 chains of MSPC slightly decrease it. The caveat is that all lipids must be ideally mixed within the main DPPC bilayer lipid. Initially, we set the host lipid as DPPC. This is the main component that establishes the transition temperature at about $41.3^{\circ} \mathrm{C}$. The actual pore forming component, MSPC lysolipid, is set at almost $10 \mathrm{~mol} \%$ (and slightly lowers the $\mathrm{T}_{\mathrm{m}}$ ). We then set the DSPE-PEG ${ }^{2000}$ lipid concentration (necessary for long circulation half life and to help stabilize the nanopores through which the drug is released) at $3.8 \mathrm{~mol} \%$ (slightly raises $T_{m}$ ). In the clinical formulation [74], the rate of release of an encapsulated drug like doxorubicin is enhanced to 10 times greater than that of the pure DPPC bilayer at its phase transition temperature by the inclusion of $\sim 10 \mathrm{~mol} \%$ MSPC lysolipid and $3.8 \mathrm{~mol} \%$ PEG-lipid.

\section{PRECLINICAL AND CLINICAL STUDIES}

The formulation of ThermoDox $^{\circledR}$, the commercial doxorubicin-loaded version of the LTSL, owes a tremendous amount to the researchers and liposomes that came before it, including DaunoXome [126-128], MYOCET [129, 130], OncoTCS [131, 132], and Doxil ${ }^{\otimes}$ [133-135]. As discussed above, lipid compositions, loading and retaining drug in the liposomes, reducing free drug toxicity, extending circulation time by evading the body's defenses, and promoting tumor accumulation through in vitro and in vivo testing have all been established. The main remaining function that had not been effectively addressed was triggered drug release. For distant metastases, this is still an issue to be solved, but several groups are making attempts, most notably, Jorgensen and Mouritsen et al., exploring a lipase-sensitive formulation [136-138]. LTSL and its heat trigger are designed to address local disease and more effective drug delivery to solid tumors.

In this second part of the review, we will begin with a brief introduction that expands on Fig. (2) describing attempts to use non-temperature-sensitive and temperaturesensitive liposomes with hyperthermia. This will be followed by a more in-depth discussion of the drug delivery issues that hyperthermia specifically addresses, the results of preclinical studies on anti-tumor effects, and imaging modalities that have led us to a new paradigm in drug delivery due to our recent observations on the microvascular effects of LTSLdoxorubicin. 
In this section, the review will address:

3.1. Rationale for Using Liposomes and Hyperthermia (Non-Temperature and Temperature-Sensitive)

3.2. Hyperthermia and Drug Delivery

3.3. Preclinical Studies: Anti-tumor Effects

3.4. Preclinical Studies: Imaging Modalities

3.5. A New Paradigm in Drug Delivery

3.6. Microvascular Effects of LTSL-Doxorubicin

3.7. Clinical Progress (Non-Temperature and Temperature-Sensitive)

3.8. Non-oncologic Applications of TemperatureSensitive Liposomes

3.9. Clinical Perspective on Cancers that would Benefit from Hyperthermia

3.10. Important Considerations for LTSL-Drug - Device Combinations

\subsection{Rationale for Using Liposomes and Hyperthermia}

\subsubsection{Non Temperature-Sensitive Liposomes}

While many non-temperature-sensitive liposomes have been developed and some are currently being used in the clinic, only Evacet and Doxil ${ }^{\circledR} /$ Caelyx have been combined with hyperthermia in the clinic. Here we explain the rationale behind using this combination, with the results of Doxil ${ }^{\circledR}$ hyperthermia clinical studies being described below in the Clinical Progress section.

As discussed above, it has long been known that tumor vasculature can be hyperpermeable [32], and especially in animal models, implanted tumors have been characterized to be leaky with enlarged endothelial pores. This leakiness has been deemed critical for allowing liposome accumulation by the Enhanced Permeability and Retention (EPR) Effect.

Previous work has shown differential permeability for tumor vasculature. Yuan et al. [139] observed a range of permeability to BSA in four different tumor models, R3230AC (rat mammary adenocarcinoma), MCaIV (mouse mammary carcinoma), U87 and HGL21 (human malignant astrocytomas). Hyperpermeability was observed in three of these tumor systems ranging from $1.7-3.8 \times 10^{-7} \mathrm{~cm} / \mathrm{s}$, while HGL2 1 showed low permeability $\left(0.11 \times 10^{-7} \mathrm{~cm} / \mathrm{s}\right)$. In a similar R3230AC model, Wu et al. [38] observed tumor vascular permeability for PEG-liposomes $\left(3.42 \times 10^{-7} \mathrm{~cm} / \mathrm{s}\right)$ and conventional liposomes $\left(1.75 \times 10^{-7} \mathrm{~cm} / \mathrm{s}\right)$. Using a human tumor (SKOV-3 ovarian carcinoma) xenograft grown in mouse window chambers, Kong et al. $[40,51]$ showed that while liposomes were unable to extravasate into the tumorinterstitium under normothermic conditions, hyperthermia enabled extravasation of liposomes of all sizes, but the magnitude of this hyperthermia-induced extravasation was inversely proportional to particle size. At normothermia $\left(34^{\circ} \mathrm{C}\right.$ ), the pore cutoff size was between 7 and $100 \mathrm{~nm}$ (i.e. between that of albumin that did extravasate and liposomes that did not), and at $42^{\circ} \mathrm{C}$, the pore cutoff size was increased to $>400 \mathrm{~nm}$, allowing for the delivery of all tested nanoparticles to the tumor interstitium to some degree. With hyperthermia, the $100 \mathrm{~nm}$ liposome experienced the largest rela- tive increase in extravasation from tumor vasculature, an increase of 1.6 and 3.5 times more extravasation compared to the 200 and $400 \mathrm{~nm}$ liposomes, respectively. This observation was tissue specific as hyperthermia did not enable extravasation of $100 \mathrm{~nm}$ liposomes from normal vasculature. Although these studies were performed in xenograft models, similar observations of hyperthermia enhancing liposome extravasation have been made in spontaneous tumors in domestic felines. Matteucci et al. [52] conducted a study on the accumulation of non-temperature-sensitive technetium-99mlabeled liposomes in feline fibrosarcomas after hyperthermia and were able to show that hyperthermia increased the accumulation of liposomes in these tumors. From timeaveraged (200 minutes) aorta-normalized tumor counts (hyperthermic/normothermic), they observed a time-averaged range of 2-13-fold increase in liposome accumulation in the tumor under hyperthermic conditions than under normothermic $\left(34^{\circ} \mathrm{C}\right)$ conditions. Moreover, there was a rise in intratumoral counts over time (ranging from 200 to 1000 minutes) following the injection indicating that the accumulation of liposomes out of the blood stream and into the tumor continued even after conclusion of the 60 minutes of heating. Thus, the enhancement of vascular permeability continues following the duration of hyperthermia.

Kleiter et al. [140] used radiolabeled liposomes in combination with Doxil ${ }^{\circledR}$ in a rat fibrosarcoma model to track liposome delivery after hyperthermia. Increased doxorubicin levels and increased radioactivity were observed at the site of the tumor after hyperthermia treatment compared to the nonheated controls, and a significant correlation was found between doxorubicin concentration and radiolabeled tracer. These results further confirmed the enhancement of extravasation both during and after hyperthermia. It has been the conventional paradigm that the mechanism of liposomal drug accumulation in interstitial tumor tissue occurs by the EPR effect. This paradigm relies on the difference in permeability between normal and tumor vasculature. Shown later, Fig. (14a and b) depict this EPR effect. If the tumor vasculature is sufficiently leaky, liposomes that have long circulation half-lives should be able to extravasate and accumulate within the tumor tissue.

In animal models, it has been found that at normal body temperature $\left(37^{\circ} \mathrm{C}\right)$ tumor vasculature has variable permeability depending on the type of tumor. There is no evidence that we are aware of that has measured the vascular permeability in tumors in humans. Mild hyperthermia increases vascular permeability of even impermeable vessels, and therefore has the potential to enhance long circulating nanoparticle extravasation. Despite the dramatic enhancement of liposomal drug accumulation in tumor interstitium by the use of hyperthermia, traditional and PEG-liposomes are designed to entrap their drug in order to achieve reduced toxicity in the delivery phase of treatment. With such products, the full potential of hyperthermia use is not achieved due to a lack of liposomal drug release. To retain drug, evade the body's defenses, and accumulate in tumors is not enough to deliver bioavailable drug to tumors. The ideal drug delivery vehicle should also possess a trigger allowing for the rapid and complete release of free drug within the tumor tissue, which is exactly what the temperature-sensitive liposome provides. 


\subsubsection{Temperature-Sensitive Liposomes}

Following Yatvin's experiments in E.Coli [23], Weinstein et al. [141] encapsulated the chemotherapeutic methotrexate and performed the first in vivo study with temperature-sensitive liposomes. Drug levels were measured in the tumor after treatment, and researchers observed significantly higher drug levels in the mice treated with the combination of hyperthermia and methotrexate temperaturesensitive liposomes compared to free drug and normothermia controls. These observations were a milestone in cancer research and have set the stage for the progress in temperaturesensitive liposomal research and applicability.

As previously mentioned, the earlier liposomes had several disadvantages described in the previous section that have since been altered in current liposome formulations. Briefly, temperatures greater than $42^{\circ} \mathrm{C}$ were required for content release, and once the $T_{m}$ had been reached, the release was slow, requiring 15 minutes to release only $40 \%$ of the contents. The new and improved LTSL that Needham and Dewhirst have developed $[46,66,73]$ have lower transition temperatures $\left(39.5-42^{\circ} \mathrm{C}\right)$ and more rapid release kinetics.

The following sections will discuss the delivery, efficacy, and imaging modalities for these LTSLs.

\subsection{Hyperthermia and Drug Delivery}

Optimal hyperthermic temperature during treatment is key to LTSL drug delivery (Fig. 11). First, the appropriate temperature $\left(T_{m}\right)$ must be reached for effective drug release. It should be noted that the elevated temperature also affects blood flow which in turn affects drug delivery. Mild hyperthermic temperatures $\left(40-43^{\circ} \mathrm{C}\right)$ increase blood flow, vascular permeability, and therefore extravasation. These effects are not observed at temperatures below this mild hyperthermia threshold, and temperatures above this threshold can result in vascular occlusion and hemorrhage, resulting in decreased blood flow and drug delivery. It is therefore critical to target $40-43^{\circ} \mathrm{C}$ when designing clinical hyperthermia protocols that aim to maximize the release and tumor tissue penetration of encapsulated drugs.
The order in which administration occurs generates several issues in using the combination treatment of LTSLs and hyperthermia. A variety of chemotherapeutic drugs including taxol, cisplatin, and doxorubicin have been shown to interact synergistically with hyperthermia, but the order in which the drug and heat are administered can have an effect on this interaction [50]. For example, Leal et al. [142] demonstrated that in vitro, cancer cells (MCF7) are protected from the cytotoxic effects of the drug taxol when mild hyperthermia $\left(43^{\circ} \mathrm{C}\right)$ is given at the beginning or in the middle of a 24 hour incubation with the drug when compared to hyperthermia being given at the end of taxol treatment. Cisplatin, on the other hand, interacts synergistically with hyperthermia $\left(42^{\circ} \mathrm{C}\right)$ when given before, during, or after heat treatment [143]. For example, Hahn [144] calculated the ratio of dose required to achieve $50 \%$ cell survival at $37^{\circ} \mathrm{C}$ to the dose required to achieve the same survival at a different temperature, or the thermal dose-modifying factors (TDMF). At $41^{\circ} \mathrm{C}$, the TMDF for cisplatin is 2.7 , and at $43^{\circ} \mathrm{C}$ it increases to 8.2. These synergistic interactions are of importance regarding temperature-sensitive liposome and hyperthermia treatment, and so choosing the drug for encapsulation and for hyperthermia-enhanced treatment is crucial to clinical success.

The order in which hyperthermia and LTSL administration occurs is critical in achieving clinical efficacy, because hyperthermia triggers the release of the drug and also increases vascular perfusion and permeability. With LTSLdoxorubicin co-loaded with manganese, a MRI contrast agent, Ponce et al. [45] showed that doxorubicin will accumulate at a five times faster rate $(9.8 \mathrm{vs} .1 .8 \mu \mathrm{g} / \mathrm{min})$, and at almost double the concentrations in the tumor (15.1 vs. 8.0 $\mathrm{ng} / \mathrm{mg}$ ) when the LTSL is administered during hyperthermia treatment compared to administering the drug before heating. Furthermore, better anti-tumor effects were observed when drug was administered during heating (discussed in more detail in the Imaging Modalities section). LTSL administered during hyperthermia yielded a median time for tumors to reach five times their pre-treatment volume of 34 days compared with 18.5 days for LTSL administration before hyperthermia, and 22.5 days for a split dose of LTSL before and

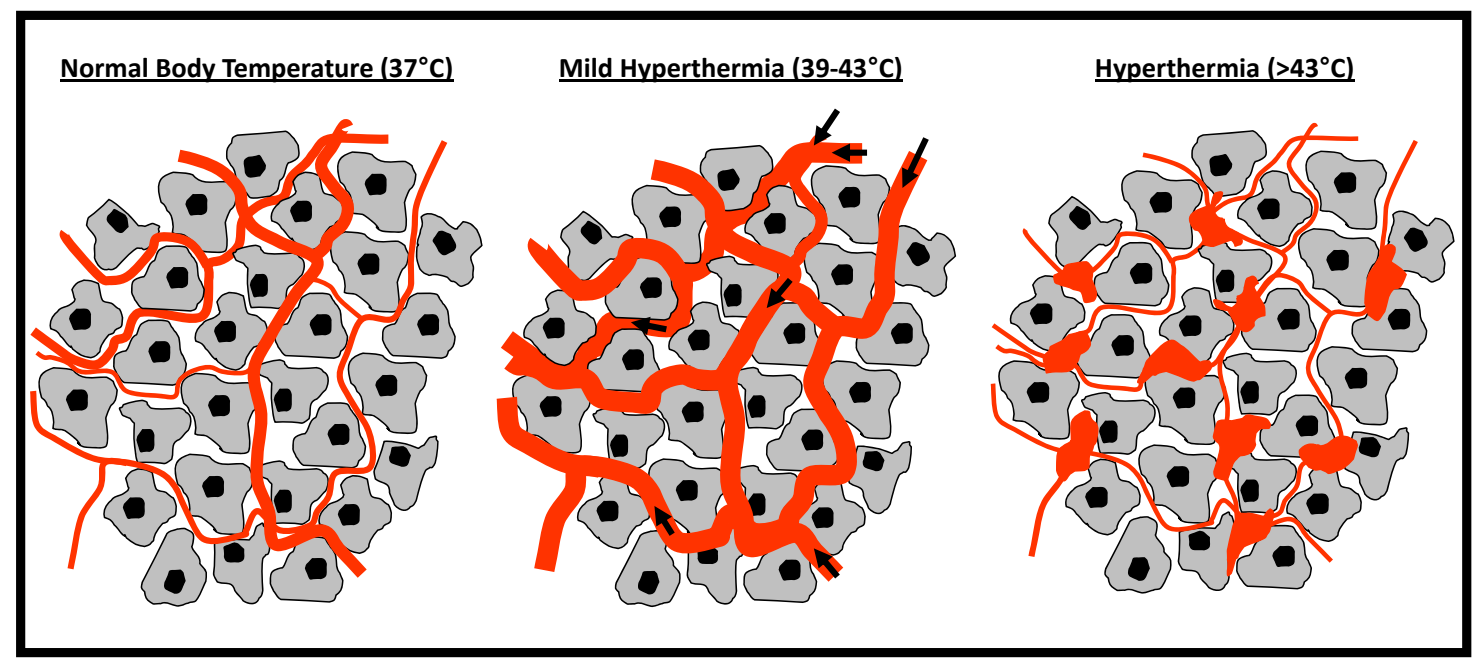

Fig. (11). Temperature effects on tumor vasculature. Mild hyperthermia increases blood flow (ideal for drug transport) whereas hyperthermia temperatures above $43^{\circ} \mathrm{C}$ result in hemorrhage, which may reduce or cease blood flow, hampering drug delivery. 
during hyperthermia. This is not a difference between hyperthermia and normothermia, but a difference between administering drug after the tumor has been warmed to the mild hyperthermic temperature of $42^{\circ} \mathrm{C}$ followed by LTSL administration, versus heating 15 minutes after LTSL administration. Thus, preheating the tumor prior to drug administration is essential in order to achieve maximal efficacy in the clinic.

Kong et al. [46] demonstrated that when combined with hyperthermia the LTSL-doxorubicin formulation significantly increases tumor drug concentration compared to free drug (20-30 times) and other liposomal formulations. The increased drug delivery was confirmed via measuring doxorubicin fluorescence in the tumor tissue as well as by measuring total doxorubicin content in the tissue bound to DNA/RNA. Kong et al. [46] observed increased tumor drug levels after only 1 hour of treatment. While free drug was virtually absent from the tumor tissue taken after the 1-hour treatment, LTSLs deposited 20-30 times more doxorubicin in the tumor tissue and 5 times more than a Doxil ${ }^{\mathbb{B}}$-like formulation.

Yarmolenko et al. [75] showed differences in drug accumulation across 5 different tumor types after treatment with LTSL-doxorubicin with or without hyperthermia. As shown in Fig. (12), hyperthermia enhances drug delivery in all tumor types by $6-15$ times compared to the normothermia controls. When treatment groups were compared within the same tumor type, a significant correlation between tumor doxorubicin concentration and greater anti-tumor effect was observed, but in the comparison across tumor types, accumulated drug concentration did not correlate with outcome. In vitro doubling times for all tumor cell lines were assessed, and this endpoint was significantly correlated with median tumor growth time. Yarmolenko et al. [75] concluded that in this model system, response is more dependent upon tumor growth rate; the slower growing tumors had increased complete regressions and the longest tumor growth delays. Unfortunately, free doxorubicin was not used as a control in this study. Comparisons of both drug delivery and anti-tumor response with free drug with and without heat across the tumor types may have been useful in the correlations. Of course, as discussed in more detail below, if drug release in the blood stream effectively killed endothelial cells, we might not expect a correlation with actual tumor drug accumulation, since variations in the degree to which the released doxorubicin could initiate vascular shut down, as observed by Chen et al. [101, 145], could conceivably account for the data. Additional studies with free drug comparisons have been done but are currently unpublished. We will discuss the anti-tumor effect of temperature-sensitive liposomes in more detail in the following section.

\subsection{Preclinical Studies: Anti-Tumor Effects}

Doxorubicin-and cisplatin-loaded liposomes make up the majority of the temperature-sensitive liposome literature [46, $55,66,68,75,84]$. Other drugs including taxol [146], melphalan $[147,148]$, methotrexate $[141,149]$, plumbagin [150], dacarbazine [151], mitomycin C [152] and tumor necrosis factor [153], have also been encapsulated (see review by Kong and Dewhirst [154]). Currently only the LTSLdoxorubicin is in clinical trials, but an optimized formulation for cisplatin and other drugs may be possible for the near future.

The first cisplatin temperature-sensitive liposome was described in 1981 by Yatvin et al. [55]. Although the liposome half-life was relatively short compared to today's PEG-liposome formulations, $t_{1 / 2}$ was only $\sim 60$ minutes for their unilamellar liposomes, it was much longer than that for free drug, $t_{1 / 2}=\sim 2$ mins. Therefore they were able to show a longer circulating time of the encapsulated cisplatin with the liposome formulation compared to free drug. Along with enhanced tumor growth delay, they observed higher cisplatin concentrations in the tumor ( $8.2 \%$ of injected dose) in the group treated with liposomes and hyperthermia compared to the free drug $(2.4 \%)$ and liposomes and normothermia (4.4\%) controls. A high rate of liposomal uptake and accumulation (much higher than observed in the tumors) was also observed in the liver. As a result, as was the case for many of the early, pre-PEG-liposome formulations, due to the toxicity issues the authors felt this formulation was not ready for clinical application. Woo et al. [68] have recently developed a formulation for cisplatin temperature-sensitive liposomes. Under normothermic conditions $\left(37^{\circ} \mathrm{C}\right)$, this formulation releases around $50 \%$ of its cisplatin contents over the course of 1-1.5 hours and was susceptible to cisplatin leakage compared to other liposomal formulations. Because of the known synergism with hyperthermia and high degree of cytotoxicity, drugs like cisplatin seem to be ideal drugs to load into liposomes, but the issue of stability in plasma needs to be addressed before moving into other animal models.

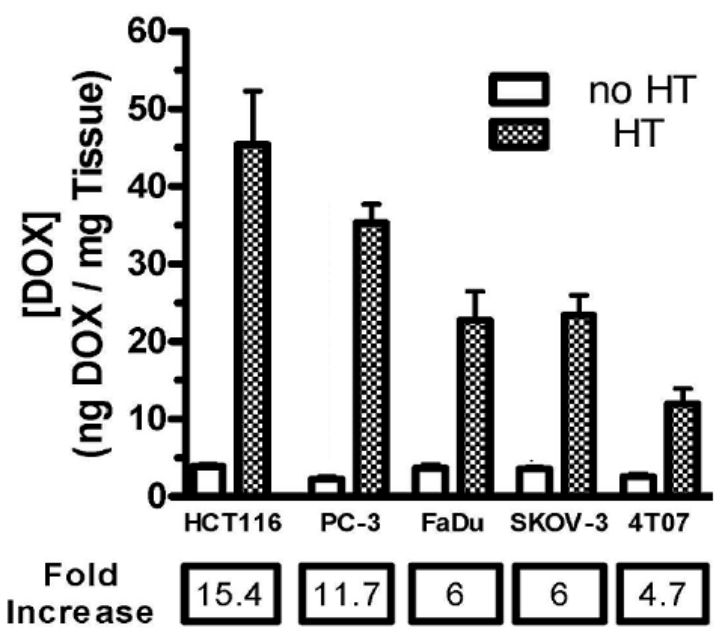

Fig. (12). A comparison of tumor doxorubicin concentrations 1 hour after LTSL-doxorubicin treatment with or without hyperthermia (HT). Hyperthermia enhances drug delivery and tumor accumulation. Adapted with permission from Informa Healthcare: [International Journal of Hyperthermia] [75], copyright (2010).

Needham et al. [66] and Kong et al.[46] reported on the first LTSL formulation that contained doxorubicin. When given intravenously, combined with local heating of a tumor bearing leg, this formulation induced greater growth delay compared to saline and free drug controls and other liposomal formulations. All tumors were locally controlled up to 60 days after the "treatment", heating the tumor to $42^{\circ} \mathrm{C}$ and injecting the LTSLs while heating continued for 1 hour [66]. Normothermic saline control tumors rapidly progressed to 5 
times tumor volume in 10 days, and free drug only extended this by 2 days. The combination of hyperthermia and free drug increased growth delay to 22 days before implanted tumors reached 5 times tumor volume. A Doxil ${ }^{\circledR}$-like liposome showed tumor responses, but all tumors grew back to 5 times tumor volume by day 35 . Thus, even though the hyperthermia plus LTSL "treatment" was only for 1 hour, the LTSL was much more effective than the Doxil ${ }^{\circledR}$-like formulation. It was this result that revealed that drug release was a crucial feature of the LTSL. Although Doxil ${ }^{\circledR}$ might extravasate in this model, the relatively slow leakage of drug would hamper its potential efficacy.

While the first experiment in the $\mathrm{FaDu}$ tumor showed such dramatic results, we wanted to test the system in additional cancer cell lines, 4T07 (murine mammary carcinoma), HCT116 (human colon carcinoma), FaDu (human squamous cell carcinoma), PC-3 (human prostate adenocarcinoma), and SKOV-3 (human ovarian carcinoma) [75]. The tumor growth delay results are shown in Fig. (13). The combination of hyperthermia and LTSL-doxorubicin was the most efficacious across all tumor types, but as depicted in the figure, the responses varied across tumor type. Temperature-sensitive liposome efficacy is hypothesized to be due to both antivascular and anti-tumor effects, therefore tumor cell kill could depend on a combination of factors including the response of the cancer cell line and the endothelial cells to the drug and the vascularity and heatability of the implanted tumor.

Recently, Palmer et al. [155] monitored tumor physiology during hyperthermia and LTSL-doxorubicin treatment with non-invasive optical spectroscopy in nude mice bearing SKOV3 ovarian tumor xenografts. Specifically, the method can measure total hemoglobin, hemoglobin saturation and fluorescence intensity of doxorubicin, which is related to blood volume, extent of hypoxia and drug concentration, respectively. The optical data demonstrated that tumors with better oxygenation and higher blood flow responded better to LTSL-doxorubicin, whereas the more hypoxic tumors exhibit a shorter time to failure (defined as time to 3 times treatment volume). The doxorubicin fluorescence measurements demonstrated that the combined hyperthermia and LTSL-doxorubicin treatment resulted in 12- to 15- fold increase in drug accumulation in tumor compared to free drug \pm hyperthermia or LTSL-doxorubicin without hyperthermia. These results are consistent with prior direct measurements of drug levels using HPLC methods [46]. While positive anti-tumor effects were observed with LTSL-doxorubicin with hyperthermia, these data do suggest that hypoxic tumor regions may be resistant to of the enhanced drug delivery afforded by LTSL-doxorubicin. It has been reported previously that doxorubicin is less effective under hypoxic conditions [156], so it is not surprising to see resistance to LTSLdoxorubicin in this condition. These results point to the need to consider multi-modality therapies that combine LTSLdoxorubicin with agents that can kill hypoxic cells.

\subsection{Preclinical Studies: Imaging Modalities}

Temperature-sensitive liposomes can also be used as an imaging modality (reviewed in Lindner et al. [157]). They have been loaded with contrast agents and have been investigated over the last decade for both imaging thermometry and drug delivery (location and accumulation). This imaging system utilizes magnetic resonance imaging (MRI) to detect contrast agents such as gadolinium or manganese that have
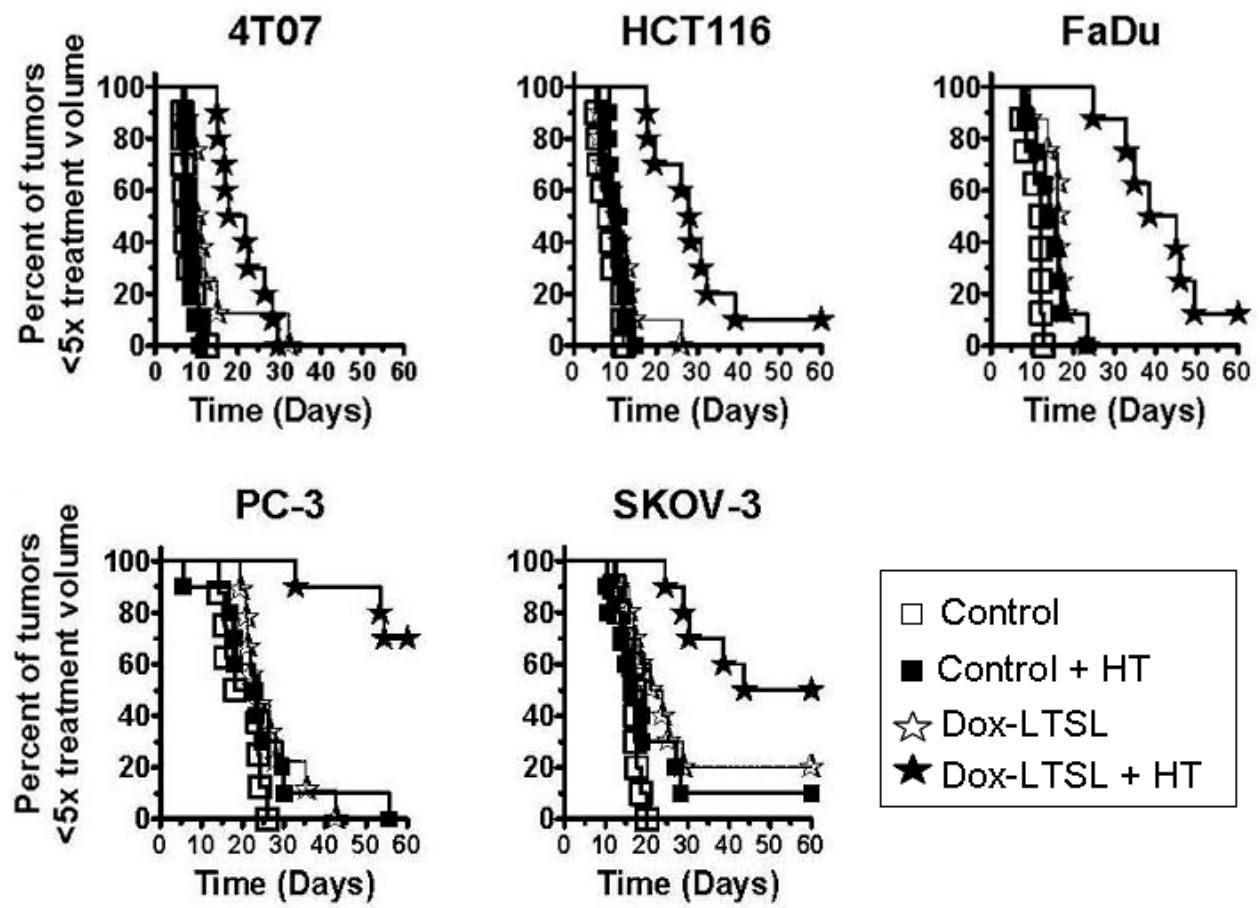

Fig. (13). A tumor growth delay study comparing the efficacy of LTSL-doxorubicin (Dox-LTSL) with or without hyperthermia (HT) in five different cancer cell lines, 4T07 (murine mammary carcinoma), HCT116 (human colon carcinoma), FaDu (human squamous cell carcinoma), PC-3 (human prostate adenocarcinoma), and SKOV-3 (human ovarian carcinoma). Kaplan-Meier plots for each tumor type are provided where the percent survival is defined as the percentage of animals with a tumor volume less than five times the original tumor volume. Adapted with permission from Informa Healthcare: [International Journal of Hyperthermia] [75], copyright (2010). 
been encapsulated in temperature-sensitive liposomes. Examples of these liposomes and their formulations are provided below in Table 2 .

MRI contrast agents for imaging have been successfully encapsulated into temperature-sensitive liposomes. For example, Zhaowuet al. [158] studied the preparation and properties of tegafur magnetic temperature-sensitive liposomes. Wang et al. [159] developed DPPGOG based temperaturesensitive liposomes loaded with the ${ }^{1} \mathrm{H}$ MR $T_{1}$ contrast agent OMINSCAN $^{\mathrm{TM}}$ (gadodiamide), and contrast agent release and MR characterization was assessed in an in vivo tumor model by Peller et al. [160]. Terreno et al. [161] prepared liposomes loaded with paramagnetic lanthanide-based complexes as $T_{2}$-based contrast media. Fossheim et al. [162] proposed that the temperature response of liposomal gadolinium diethylenetriaminepentaacetic acid bis(methylamide) (GdDTPA-BMA) is linked to the phase-transition properties of the liposome carrier. Langereis et al. [163] developed a combined ${ }^{1} \mathrm{H}$ CEST (chemical exchange saturation transfer) and ${ }^{19} \mathrm{~F}$ MRI probe-loaded temperature-sensitive liposomes. The CEST-based contrast enhancement allows for drug carrier localization and the MR signal allows for drug release quantification.

Co-encapsulation of drug with contrast agent has been the focus of recent temperature-sensitive liposome studies. Recently de Smet et al. [164] co-encapsulated doxorubicin and $\left[\mathrm{Gd}(\mathrm{HPDO} 3 \mathrm{~A})\left(\mathrm{H}_{2} \mathrm{O}\right)\right]$ and were able to demonstrate in vitro that this co-encapsulation does not alter the doxorubicin release kinetics or compromise drug encapsulation. Several studies have also used temperature-sensitive liposomes coloaded with doxorubicin and the MRI contrast agent manganese $[45,165,166]$. Viglianti et al. [166] confirmed both in vitro and in vivo a linear relationship between doxorubicin concentrations determined via MRI ( $T_{1}$ relaxation time) and concentrations measured invasively (doxorubicin fluorescence in the tissue and doxorubicin in tumor extractions). Ponce et al. [45] utilized these same liposomes to examine drug delivery differences when manipulating the timing of drug administration and hyperthermia. Intratumoral drug distribution and concentration could be controlled by these manipulations and monitored via MRI. The MRI-based doxorubicin concentration measurements were significantly associated with treatment outcome. These studies and imaging systems could have a profound effect in the clinic offering a real-time evaluation of drug delivery and potentially serving as a predictor of patient outcome. These studies currently remain in the preclinical stage but have definite translational potential for the clinic.

\subsection{New Paradigm of Temperature-Sensitive Liposome Drug Release}

Although the EPR effect may be the main delivery mechanism for non-temperature-sensitive liposomes such as Doxil $^{\circledR}$, a new paradigm in temperature-sensitive liposomal drug release is emerging (Fig. (14c)). Kong et al. [46] suggested that the release mechanism that results in increased drug levels relative to other liposomal formulations is drug release within the tumor vasculature as opposed to within the interstitial space. Recent preliminary data collected by our lab in collaboration with Lindner's group supports the hypothesis of intravascular drug release from LTSL. Using skin-fold window chambers containing tumors, confocal microscopy was used to show that doxorubicin is released from LTSL within the vasculature (intravascular release), and then extravasates into and accumulates within the tumor tissue down its own concentration gradient. Needham et al. [66] showed that LTSL-doxorubicin can release contents within tens of seconds, so it is logical that the drug release could occur within the vasculature before liposomes extravasate into the tumor tissue. This would result in an increased drug concentration within the vessels resulting in a concentration gradient that drives the drug deeper within the tumor tissue, further away from the blood vessels, ultimately exposing the tumor and tumor microenvironment to elevated levels of drug.

\subsection{Microvascular Effects of Doxorubicin-LTSL}

Tumor vascularity varies between tumor type and individuals, and more vascularized (or better perfused) tumors may allow for more efficient drug delivery, where a higher drug concentration can reach the site of the tumor. Chen et al. [101, 145] assessed vascular effects of hyperthermia and LTSL-doxorubicin in two different tumor models, a human

Table 2. Examples of Studies Using Temperature-Sensitive Liposomes as an Imaging Modality

\begin{tabular}{|c|c|c|c|c|}
\hline Author & Liposome Content & Liposome Formulation & Model System & Reference \\
\hline Terreno et al. & Lanthanide-based complexes & DPPC:DSPE-PEG ${ }^{2000}$ :cholesterol & In vitro and in vivo & {$[161]$} \\
\hline Langereis et al. & $\begin{array}{l}{\left[\mathrm{Tm}(\mathrm{hpdo} 3 \mathrm{a})\left(\mathrm{H}_{2} \mathrm{O}\right)\right] \text { (chemical shift }} \\
\text { agent) and } \mathrm{NH}_{4} \mathrm{PF}_{6}\left({ }^{19} \mathrm{~F} \text { MRI probe }\right)\end{array}$ & $\begin{array}{l}\text { MPPC/DPPC/DPPE-PEG }{ }^{2000} \\
10: 90: 4\end{array}$ & In vitro & {$[163]$} \\
\hline de Smet et al. & $\begin{array}{l}{\left[\mathrm{Gd}(\mathrm{HPDO} 3 \mathrm{~A})\left(\mathrm{H}_{2} \mathrm{O}\right)\right] \text { and doxoru- }} \\
\text { bicin }\end{array}$ & $\begin{array}{l}\text { DPPC:MPPC:DPPE-PEG }{ }^{2000} \\
(86: 10: 4)\end{array}$ & In vitro & [164] \\
\hline $\begin{array}{l}\text { Viglianti et al. and Ponce } \\
\text { et al. }\end{array}$ & Manganese and doxorubicin & $\begin{array}{l}\text { DPPC:MSPC:DSPE-PEG } \\
(90: 10: 4)\end{array}$ & In vitro and in vivo & {$[45,165,166]$} \\
\hline
\end{tabular}




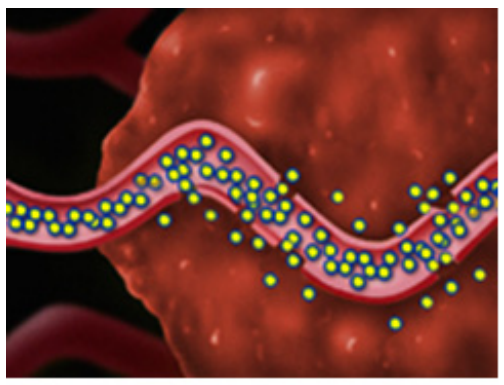

(a) Leaky tumor vessels (at $37^{\circ} \mathrm{C}$ )

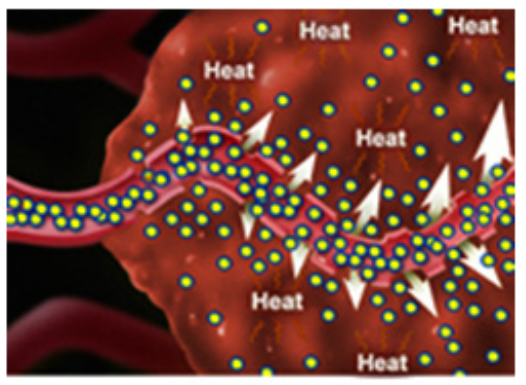

(b) Heat enhances vascular permeability $\left(39^{\circ}<\mathrm{T}<42^{\circ} \mathrm{C}\right.$ )

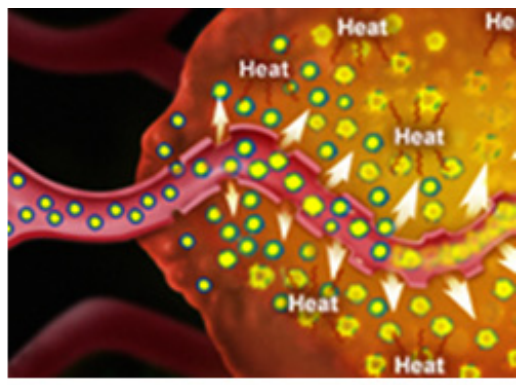

(c) Thermally-mediated release (at $39^{\circ}-42^{\circ} \mathrm{C}$ )

Fig. (14). Comparative paradigms for liposomal drug delivery. Shown are (a) Non-temperature sensitive liposomes (blue/yellow circles) preferentially extravasate from pores in tumor vessel walls; this is the standard EPR effect in normothermic systems, (b) hyperthermia increases tumor vessel pore size and thus increases non-temperature-sensitive liposome extravasation, and (c) hyperthermia can trigger drug (yellow) release from LTSL in the tumor vessel at mild hyperthermic temperatures. (c) depicts intravascular drug release and deeper penetration in to tumor tissue, representing a new paradigm of thermosensitive drug release.

pharyngeal squamous carcinoma cell line $(\mathrm{FaDu})$ and a murine mammary carcinoma cell line (4T07). A decrease in tumor blood flow ( $\sim 99 \%$ in $\mathrm{FaDu}$ and $85 \%$ in $4 \mathrm{~T} 07)$ and microvessel density (78\% in $\mathrm{FaDu}$ and $69 \%$ in $4 \mathrm{~T} 07)$ was observed after treatment but to a lesser extent in $4 \mathrm{~T} 07$ tumors. A key difference between these tumor models is vascular permeability. Hyperthermia increased microvascular permeability by 9 -fold in $\mathrm{FaDu}$ tumors, while a nonsignificant increase in permeability was observed in 4T07 tumors potentially resulting in a lower drug concentration at the site of the tumor. Vascular permeability plays a key role in antivascular effects and potentially in anti-tumor effectiveness of the drug, in situations where extravasation is required for efficacy. These results suggest that doxorubicin is acting on the tumor vasculature as well as the tumor cells and is further evidence and support for an intravascular release from LTSLs.

\subsection{Clinical Progress}

As of summer 2010, 449 liposomal-based clinical trials are listed on the NIH www.ClinicalTrials.gov site [167]. These include recruiting, ongoing, terminated, and completed trials. Of these, 348 trials are for oncologic treatment, and 22 involve hyperthermia treatment. Based on these numbers, it is obvious that there is a tremendous opportunity for increased liposome use in the clinic.

\subsubsection{Non-Temperature-Sensitive Liposomes}

There are currently 220 clinical trials listed for treatment with liposomal doxorubicin [167]. Although many of these trials are completed, only four have results posted for the public. These trials were for the treatment of multiple myeloma, metastatic breast cancer, or epithelial ovarian cancer, and Doxil ${ }^{\mathbb{B}}$ was either used alone or in combination with other chemotherapeutic agents, depending on the trial. The randomized trial for newly diagnosed multiple myeloma patients compared the efficacy of Thalidomide and Dexamethasone vs. the addition of Doxil ${ }^{\circledR}$ to the same treatment [168]. There was no significant difference between the two groups in terms of overall response, complete response, or time to first response.

A phase III study conducted by Orlowski et al. [169] in relapsed or refractory multiple myeloma patients demon- strated that the combination of PLD and bortezomib improved time to progression compared to bortezomib alone (6.5 and 9.3 months, respectively) and also improved the 15 month survival rate ( $76 \%$ and $65 \%$, respectively). Gordon et al. [170] conducted a long-term follow-up study of a phase III trial treating recurrent or refractory epithelial ovarian cancer with either PLD or topotecan. Patients treated with PLD demonstrated prolonged survival compared to the topotecan treated patients (median survival 62.7 weeks and 59.7 weeks, respectively), and more specifically, patients with platinum-sensitive diseases showed a prolonged survival of 107.9 weeks in PLD-treated patients compared to 70.1 weeks in topotecan-treated patients. In a small study with 61 recurrent epithelial ovarian or peritoneal carcinoma patients, Alberts et al. [171] compared the efficacy of PLD combined with carboplatin to carboplatin alone. Improved response rates were observed in patients treated with carboplatin and PLD (67\%) compared to the carboplatin alone group (32\%). An additional phase III clinical study assessed the efficacy of PLD compared to the combination of doxorubicin, bleomycin, and vincristine in patients with advanced AIDS-related Kaposi's sarcoma [172]. An overall response rate of $45.9 \%$ was observed in the PLD-treated patients compared to $24.8 \%$ in patients treated with the combination chemotherapy, but survival rates did not differ between the two groups. Although adverse events were common in both groups, PLD appeared to be better tolerated. It is important to note that these studies did not directly compare PLD to free doxorubicin.

Other published clinical trials for Doxil ${ }^{\circledR}$ have shown its main benefit to be decreased toxicity. Garcia et al. [173] assessed soft tissue sarcoma patients treated with Doxil ${ }^{\mathbb{B}}$ and observed favorable toxicity profiles but a lack of increased drug effectiveness compared to free doxorubicin. The same was observed in Ellerhorst et al. [174] in metastatic melanoma patients. In advanced gynecologic patients, Isrel et al. [175] observed both favorable toxicity profile and prolonged responses in heavily pretreated patients. Because of the reduced toxicity observed with Doxil ${ }^{\mathbb{B}}$, phase I clinical trials such as Garcia et al. [176] in combination with topotecan and Iqbal et al. [177] in combination with docetaxel are working on finding the most effective combinations. As discussed earlier, despite the successes in reduced toxicity, lack 
of improved efficacy in these studies may be due to slow release of bioavailable drug within the tumor tissue in humans.

\subsubsection{Non-Temperature-Sensitive Liposomes and Hyper- thermia}

Three clinical studies have been performed using combinations of hyperthermia and non-temperature-sensitive liposomes such as Evacet and Doxil ${ }^{\circledR}$. Below are descriptions of these studies with different treatment regimes and different outcomes.

Vujaskovic et al. [178] conducted a phase I/II study on locally advanced breast cancer patients using the neoadjuvant combination therapy of paclitaxel, liposomal doxorubicin (Evacet), and hyperthermia. Patients were given four cycles of this neoadjuvant therapy followed by surgical intervention, radiation therapy, and 8 cycles of cyclophosphamide, methotrexate, and 5-fluorouracil chemotherapy. The treatment was well tolerated. A correlation with thermal dose and pathologic response was observed. The combined (partial and complete) clinical response rate and combined pathological response rate were $72 \%$ and $60 \%$, respectively. Four patients achieved a pathologically complete response, and 16 patients were eligible for breast-conserving surgery. Cumulative equivalent minutes (CEM 43) at the $10^{\text {th }}$ percentile of temperature distribution (T90) was significantly greater for those with a pathological response. The four-year disease-free survival and overall survival was $63 \%$ and $75 \%$, respectively. A potential addition to heat and liposome treatment is radiation treatment. As hyperthermia increases vascular perfusion, it serves as a way to decrease the hypoxic radioresistant regions. As shown in the previous study, this trimodal treatment may be a more effective combination.

Kouloulias et al. [179] conducted a phase I/II trial to assess the tolerability and activity of the combination of Caelyx (pegylated liposomal doxorubicin), local hyperthermia and reirradiation in patients with locally recurrent breast cancer. In this study, the combination was well tolerated and effective. A measureable response was observed in all patients, and 3 of the 15 patients underwent a clinically complete response. Caelyx was administered concurrently with radiation initially. The infusions were repeated every 4 weeks for 6 months and hyperthermia treatment followed each of the Caelyx infusions, but the time between drug infusion and hyperthermia treatment ranged between 3 and 40 hours. Their analysis showed that patients had a better response when this gap did not exceed 12 hours, and the smaller the time interval the better the response. It is important to note that the temperatures used in this study were relatively high (around $44^{\circ} \mathrm{C}$ ).

Secord et al. [180] conducted a phase I/II trial with intravenously administered Doxil ${ }^{\mathbb{B}}$ and whole abdomen hyperthermia in patients with paclitaxel- and/or platinum-resistant ovarian cancer. The MTD of hyperthermia treatment was determined in the phase I portion. Women were able to undergo 60 minutes of heating once the average vaginal and rectal temperatures had reached $40^{\circ} \mathrm{C}$ or 90 minutes after power was turned on. Seventy percent of the patients treated experienced adverse events, and the majority of these were associated with Doxil ${ }^{\mathbb{R}}$ and not hyperthermia. The majority of patients $(63 \%)$ had to terminate the study early because of progressive disease. Phase II and III clinical trials with Doxil $^{\mathbb{B}}$ in platinum-resistant ovarian cancer patients showed response rates of $12.1-26 \%$, and the addition of hyperthermia treatment in Secord et al. [180] did not increase the drug efficacy. The authors stated that Doxil ${ }^{\circledR}$ may not be the ideal chemotherapeutic drug to combine with hyperthermia for ovarian cancer treatment.

Thus, while the combination of Doxil ${ }^{\circledR}$ or Caelyx and heat in breast cancer patients seemed encouraging, the combination was not ideal for ovarian cancer patients. The difference in outcome cannot be isolated to one variable; these were different cancer types receiving different treatment regimes. One key difference among the studies is the better response observed when treating a more superficial disease as opposed to deep, whole cavity (e.g. abdomen) heating. It is also important to note that thermal dose was a key factor for the responses observed in patients in the Vujaskovic et al. [178] and Kouloulias et al. [179] studies. This emphasizes the benefit hyperthermia can have in clinical situations as well as the importance in carefully monitoring and maintaining precise temperatures during treatment.

\subsubsection{LTSLs (ThermoDox ${ }^{\circledR}$ ) and Hyperthermia}

Hauck et al. [181] described the first canine phase I trial with LTSL-doxorubicin and hyperthermia. Toxicity and drug pharmacokinetics were examined in dogs with solid tumors. One objective of the study was to determine the maximum tolerated dose. The MTD observed in this study was 0.93 $\mathrm{mg} / \mathrm{kg}$, which is slightly lower than that for free doxorubicin and Doxil ${ }^{\mathbb{R}}$ studies, $0.975 \mathrm{mg} / \mathrm{kg}$ [182] and 1.0 [183] mg/kg, respectively. The initial cohort of animals experienced anaphylactoid reactions to the drug, which led to the necessity of pretreating subsequent animals with steroids and antihistamines prior to liposomal administration. This is common for injected drug delivery systems and is now stipulated for human trials. Acute toxicities (myelosuppression) more closely resembled that of free drug. As expected because of the relatively long circulation time of the pegylated LTSLs, decreased clearance of doxorubicin was observed in the patients as compared to free drug, and tumor drug concentrations were much higher ( $\sim 10$ fold) as compared to free drug and hyperthermia studies. A response rate of $30 \%$ was observed in the patients which is higher than observed in canine phase II trials with doxorubicin.

ThermoDox ${ }^{\circledR}$ is currently being tested in two trials sponsored by the Celsion Corporation, both of which involve its use as a single agent treatment in combination with hyperthermia. The two trials are a phase I/II for chest wall recurrences of breast cancer using BSD-500 ${ }^{\mathrm{TM}}$ System [47] at nine sites in the USA where target temperatures are $40-42^{\circ} \mathrm{C}$, and a phase III trial using RF thermal ablation for treatment of non-resectable hepatocellular carcinoma [49].

The Celsion phase I clinical trial for patients with primary and metastatic tumors of the liver is now completed [48]. In this study, patients were treated with a combination of RF ablation and ThermoDox ${ }^{\circledR}$. A 30-minute infusion of ThermoDox ${ }^{\circledR}$ was given 15 minutes prior to ablation. Pharmacokinetic data from this study is provided in Fig. (15) and in Poon and Borys [184]. This was a dose escalation study used to find the MTD $\left(50 \mathrm{mg} / \mathrm{m}^{2}\right)$. They observed a statistically significant difference in the time to treatment failure 


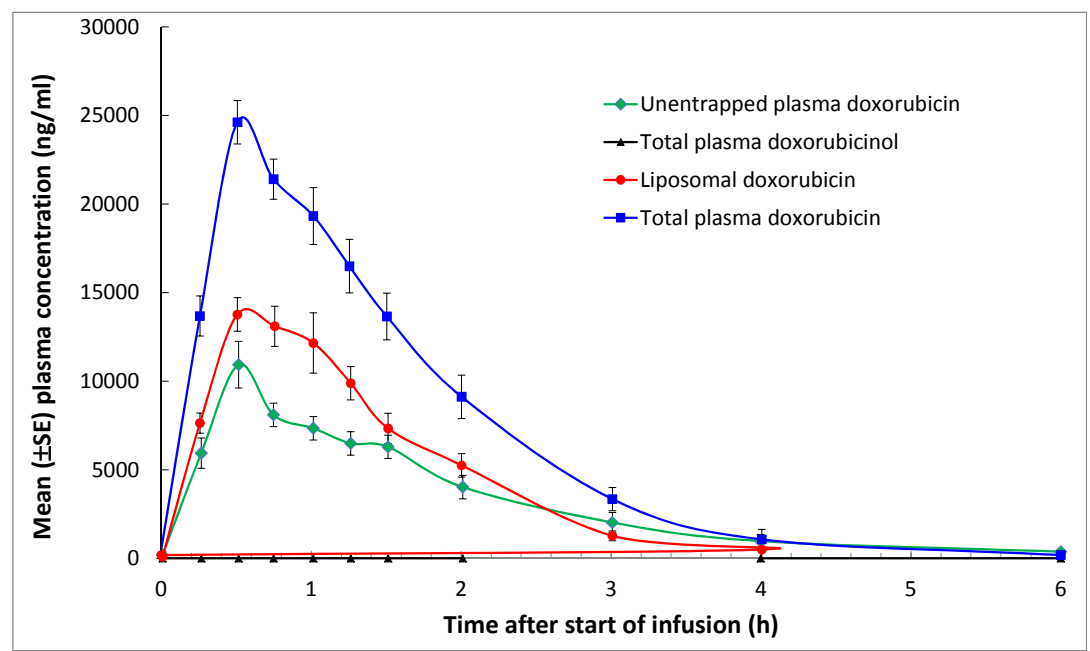

Fig. (15). Human plasma clearance of $50 \mathrm{mg} / \mathrm{m}^{2}$ ThermoDox ${ }^{\circledR}$ (Mean $+/-\mathrm{SE}$ ). Reprinted with permission [216].

between the patients receiving at least $50 \mathrm{mg} / \mathrm{m}^{2}$ and patients receiving less that $50 \mathrm{mg} / \mathrm{m}^{2}$ (374 days vs. 80 days, respectively). They concluded that the combination of RF ablation and ThermoDox ${ }^{\circledR}$ was safe and likely more efficacious than $\mathrm{RF}$ ablation alone.

As a result, Celsion is currently sponsoring a global phase III clinical trial treating hepatocellular carcinoma patients with RF ablation and ThermoDox ${ }^{\circledR}$ (NCT00617981 [49]). This trial was recently recognized by the Consensus Recommendations of the National Cancer Institute Clinical Trials for hepatocellular carcinoma [185]. The accrual goal is 600 patients at 77 clinical trial sites across 11 different countries. To date, (08/2010) approximately half of this study population has already been enrolled, and they expect no issues with recruiting the additional patients [105].

Celsion is completing a phase I and initiating a phase II trial where ThermoDox ${ }^{\circledR}$ and hyperthermia are being used in combination to treat recurrent chest wall disease in breast cancer patients (NCT00826085 [47]). A prior phase I study was conducted at Duke University; a manuscript is being prepared for submission. Because of the broad spectrum of anti-tumor efficacy of doxorubicin, ThermoDox ${ }^{\circledR}$ has the potential to be used to treat multiple other cancer types in the future.

In combination with Philips Healthcare, a manufacturer of HIFU systems, Celsion is also interested in initiating clinical trials to assess the use of HIFU in combination with ThermoDox ${ }^{\circledR}$ in metastatic bone cancer patients. Additionally, Celsion is looking into the treatment of pancreatic cancer with ThermoDox ${ }^{\mathbb{B}}$, but these are still in the preclinical stages of research [105].

The initial half-life of ThermoDox ${ }^{\circledR}$ is longer than that of free drug, but substantially less than that of Doxil ${ }^{\circledR}$. When considering the average duration of heating in the clinic (3060 minutes), it is clear that optimal drug delivery would be achieved if hyperthermia treatment was started prior to administration of drug. This sequence would take maximal advantage of high intravascular LTSL concentrations. The preclinical results from Ponce et al. reveal the importance of sequencing as well [45].

\subsection{Non-Oncologic Applications of Temperature- Sensitive Liposomes}

Although temperature-sensitive liposomes have been modified and optimized for cancer treatment since the late 1970s, there are other applications for this combination of heat and liposomes. Although the focus of this review is oncologic applications, examples of non-oncologic applications are described below.

One technique that has been in use since before 1990 is laser-targeted angiography (LTA) which is a technique used to visualize the vessels of the eye (reviewed by Hirata and Nishiwaki [186]). Liposomes are loaded with carboxyfluorescein and release the contents when heated/disrupted by a mild laser pulse. Similar to the LTSLs mentioned in the preclinical studies, but using a different liposome composition comprising a mixture of dipalmitoylphosphatidylglycerol and dipalmitoylphosphatidylcholine, the phase transition temperature is around $41^{\circ} \mathrm{C}$. In LTA, liposomes are injected intravenously and a laser is pulsed through the pupil. The goal is to visualize the bolus of carboxyfluorescein in the retinal or choroidal vessels. One advantage of this technique over others (e.g. injectable dye) is that the process can be repeated multiple times, as long as the liposomes are circulating in the blood stream. This technique remains in the preclinical stages (rodents and monkeys) but has the potential for both lesion detection and treatment. It has proven useful in imaging choroidal circulation in vivo [187]. The use of LTA has successfully been used to detect submacular lesions such as choroidal neovascularization in rodent models [188]. Asrani and Zeimer [189, 190] have successfully loaded the photosensitizer aluminium phthalocyanine tetrasulphonate and treated rats with laser targeted photoocclusion resulting in occlusion of vessels. Potential next steps are to use this technique in humans and to load the liposomes with drugs that have to ability to treat ocular diseases and eye infections.

The use of temperature-sensitive hydrogels has been growing in interest for several different treatment options such as drug delivery, cell encapsulation, and tissue repair (reviewed by Ruel-Gariepy and Leroux [191]). The overall idea is that fluid can be injected into the body and at the body temperature $\left(37^{\circ} \mathrm{C}\right)$ a gel matrix is formed. Tempera- 
ture-sensitive liposome based models in addition to several other models are currently being examined. These can potentially be used to fill a cavity or defect or be used to slowly release a drug [192, 193].

\subsection{Clinical Perspective on Cancers that would Benefit from Hyperthermia}

The use of LTSLs in conjunction with mild hyperthermia has shown tremendous promise, both in preclinical studies and in clinical trials that are now progressing through phase III. Thus, the review will conclude with a brief perspective on hyperthermia as an adjuvant modality for cancer treatment in the hope that the successes already achieved by this relatively underappreciated technique can now motivate the development of additional thermally-triggered chemotherapeutic delivery systems.

In 1986, Kapp [194] authored a review on the types of cancer that, based on lesion location and potential progression, would benefit from hyperthermia. Several criteria for site and disease selection for hyperthermia treatment were explained.

1) Site selection should be based on tumors in which the local control rates are poor with conventional radiation therapy and/or surgery or chemotherapy.

2) Improvement in local control of these sites should result in either higher cure rates and/or prevention of significant patient morbidity.

3) Sufficient numbers of patients should be available for protocol study.

4) 'Adequate' heating and temperature monitoring should be attainable by available technology in the tumor sites selected.

5) The tumor heating should be accomplished without excessive normal tissue toxicity or patient discomfort.

This review by Kapp assessed in detail the diseases that may benefit from adjuvant hyperthermia treatment. Based on mortality statistics, they found that local failures contribute to death in a high proportion of patients with brain, ovarian, prostate, cervical, esophageal, bladder, and head and neck cancers. They also described lesions for which local-regional recurrence and metastases are problematic and may be candidates for hyperthermia benefit such as in breast cancer, head and neck cancer (lymph node metastases), colorectal cancer (nodal metastases), bladder cancer (muscle invasive disease), and malignant melanomas (symptomatic cutaneous, subcutaneous, or superficial lymph node metastases).

A thorough review was done by Falk and Issels [195] discussing clinical trials prior to 2001 that utilized hyperthermia as an adjuvant therapy in a variety of solid tumors. They described the feasibility and effectiveness of hyperthermia treatment in multiple clinical studies in locally advanced tumors in terms of objective response rate, local tumor control, and relapse free survival. It was noted that specifically in breast cancer, head and neck tumors, cervical cancer, and glioblastoma, the addition of hyperthermia to other treatments such as radiotherapy significantly improves response and survival. This review stated that in patients receiving treatment for locally advanced superficial tumors complete responses rates with hyperthermia alone are $15 \%$, with radiotherapy alone are $35 \%$, and the combination treatment is approximately $70 \%$ [195].

Although superficial lesions, because of accessibility, seem to be an ideal target for hyperthermia adjuvant treatment, improvements in heating devices now also allow for targeting deep-seated tumors. Several heating devices have been developed to accommodate these types of cancer such as the HYPERcollar for head and neck cancer [196], superficial devices for breast cancer (Duke Breast Applicator System) [178], microwave devices for brain heating [197], and systems like the BSD-2000 for deep-seated lesions (cervical, gastrointestinal, bladder, etc) [198]. As shown in Table 3, mild hyperthermic temperatures in the range required for drug release from temperature-sensitive liposomes such as ThermoDox ${ }^{\circledR}$ are feasible in superficial and deep-seated tumors.

Table 3. Thermometry Data from Several Studies Indicating the Feasibility of Tumor Heating to a Range Sufficient for Drug Release from LTSL

\begin{tabular}{|c|c|c|c|c|c|}
\hline \multirow{2}{*}{ Author } & \multirow{2}{*}{ Tumor Type } & \multicolumn{3}{|c|}{ Thermal Data $\left({ }^{\circ} \mathrm{C}\right)$} & \multirow{2}{*}{ Reference } \\
\hline & & T90 & T50 & T10/T20 & \\
\hline Gellermann et al. & sarcoma & 40.8 & 43.6 & 46.2 & [199] \\
\hline Leopold et al. & sarcoma & 39.5 & 41.6 & 43.0 & [61] \\
\hline Wust et al. & pelvic & $39.9( \pm 0.9)$ & NR & NR & [63] \\
\hline Sherar et al. & $\begin{array}{l}\text { chest wall recur- } \\
\text { rences of breast } \\
\text { cancer }\end{array}$ & $40.5-41.4^{*}$ & NR & NR & [60] \\
\hline Milani et al. & rectal & 41.4 & 42.9 & 43.5 & {$[62]$} \\
\hline Franckena et al. & cervix & $39.8( \pm 0.55)$ & $40.6( \pm 0.55)$ & $41.1( \pm 0.31)$ & {$[200]$} \\
\hline Vujaskovic et al. & $\begin{array}{l}\text { locally advanced } \\
\text { breast }\end{array}$ & $39.6( \pm 0.86)$ & $41.0( \pm 0.92)$ & NR & {$[178]$} \\
\hline
\end{tabular}

T90, T50, T20, and T10 are the temperatures to $90 \%, 50 \%, 20 \%$, and $10 \%$ of the tumor tissue, respectively.

*A range of T90s from four different centers. 
Local control for advanced head and neck tumors remains a challenge in the clinic. Recently, Paulides et al. [196] developed a heat applicator specifically for heating the head and neck region. The HYPERcollar system was developed to provide maximum heating to the target region while minimizing heating to critical tissues such as the spinal column [196, 201]. This system uses a phased-array of 12 antennas to deliver heat while keeping the skin relatively cool with a water bolus cooling system. Clinical feasibility of the HYPERcollar was assessed in three patients with lesions in the thyroid, oropharynx, and nasal cavity [201]. Patients were treated with the combination of radiotherapy and hyperthermia. Patient outcome data was not provided, but it was concluded that the use of the HYPERcollar was practical in clinical settings and may provide better treatment quality in the future.

Hyperthermia may also be a beneficial adjuvant for breast cancer treatments. A recent meta-analysis conducted by the Early Breast Cancer Trialists' Collaborative Group assessed if the variations in local treatment that can affect the risk of locoregional recurrence could also affect mortality [202]. Individual patient data from 78 randomized treatment comparisons were examined, and the data suggested that improvements in local control would significantly reduce breast cancer mortality. As mentioned previously, it is clear from the Vujaskovic et al. [178] and Kouloulias et al. [179] studies that thermal dose plays a role in breast cancer patient outcome. Duke University has developed a heating applicator for the breast, Duke Breast Applicator System (DBAS). Treatment with this system has recently provided encouraging results [178] (see Table 3). This system involves a waterfilled cup that provides temperature control at the skin's surface and electromagnetic coupling for heating. A vest applicator for treating large, diffuse areas of chest wall disease has also been developed [203]. This microwave array applicator uses a water bolus to conform to body contours in such a way that the vest maintains contact with the skin to both prevent distortions in the microwave power deposition pattern and to prevent the skin from overheating.

Hyperthermic isolated limb perfusion is a treatment used for locoregionally metastasized malignant melanoma. A recent outcome study for patients with locoregionally metastasized malignant melanomas treated with hyperthermic isolated limb perfusion observed an overall response rate of $80.7 \%$ and a complete remission was observed in $62.8 \%$ of the patients [204]. This therapy was considered efficient and effective for patients with multiple or recurrent in-transit metastases, and long-term survival was attainable in patients that did not present with regional lymph node metastases or distant metastases. This again supports the need for local and regional control in order to achieve increased survival.

Issels et al. [205] recently published a multi-institutional phase III clinical trial for patients with localized high-risk soft-tissue sarcoma. Patients were treated with either neoadjuvant chemotherapy consisting of etoposide, infosfamide, and doxorubicin alone, or in combination with regional hyperthermia in addition to local therapy (surgery and/or radiotherapy). Local progression-free survival was the primary endpoint. Hyperthermia was administered for 60 minutes with a target tumor temperature of $42^{\circ} \mathrm{C}$ via the BSD-2000 hyperthermia system, an annular phased array system in which microwave antennas are arranged around the axis of the body. The addition of hyperthermia to chemotherapy resulted in significantly better local progression-free survival and disease-free survival than the chemotherapy alone. The main benefit was observed in patients with non-extremity tumors for which the disease-free survival rate at two years for the combined arm and the chemotherapy alone arm was 50 and $33 \%$, respectively.

Systems like the BSD-2000 can also administer deep regional hyperthermia. Fatehi et al. [198] treated patients with locally advanced cervical carcinoma with a combination of local-regional deep hyperthermia, radiotherapy, and chemotherapy to assess temperature distributions throughout the abdominal cavity. Jones et al. [206] conducted a phase I/II trial for patients with advanced, recurrent, or progressive ovarian carcinoma. Patients were treated with i.p. cisplatin, i.v. thiosulphate, and concurrent whole abdomen hyperthermia. Hyperthermia was administered with the BSD-2000 system. They observed an overall response rate of $44 \%$ even though the majority of the patients had platinum-resistant disease. This patient population has typical response rates of $10-20 \%$ following free drug administration. This suggests that hyperthermia is adding some benefit over what can be achieved with free drug.

Bladder cancer patients have also shown clinical benefits from hyperthermia treatment. Colombo et al. [207] treated patients with primary or recurrent superficial transitional cell carcinoma of the bladder with intravesical mitomycin $\mathrm{C}$ with or without hyperthermia. Hyperthermia was administered with an intravesical microwave applicator (Synergo SBTS:101-1 (Medical Enterprises, Amsterdam, the Netherlands)). Significantly fewer recurrences were observed in patients treated with the combination of mitomycin $\mathrm{C}$ and hyperthermia compared to mitomycin $\mathrm{C}$ alone. A similar study using this same intravesical system combined with mitomycin $\mathrm{C}$ in patients with multiple or recurrent transitional cell carcinoma of the bladder exhibited similar results showing a high percentage of recurrence-free patients [208]. A trial treating high-grade superficial bladder cancer with the Synergo system and mitomycin $\mathrm{C}$ was also beneficial with $62.5 \%$ of patients being recurrence-free [209].

Highly aggressive brain tumors such as glioblastomas may benefit from hyperthermia treatment with the appropriate heating device. Sneed et al. [197] compared glioblastoma patient outcome when treated with radiotherapy and brachytherapy with or without hyperthermia treatment in a randomized phase III trial. Hyperthermia treatment was administered with helical-coil microwave antennas. A survival benefit or local control was observed with the addition of hyperthermia treatment. Ultrasound methods are also available for heating brain tumors. Guthkelch et al. [210] conducted a phase I study in patients with primary malignant tumors of the brain treating with a combination of hyperthermia administered via scanned focused ultrasound (SFUS) and external beam radiation. Based on several autopsies, they determined that use of the SFUS system in combination with radiotherapy was effective in causing necrosis in glioblastoma multiforme within the heated tumor volume.

Pulsed high-intensity focused ultrasound (HIFU) may be an option for hyperthermia administration in the near future. HIFU is currently used in the clinic for ablative treatments 
(reviewed in [211]) reaching high temperatures by using continuous ultrasound exposures, but as described in Dromi et al. [212], pulsing HIFU exposures can elevate tissue temperature to more mild hyperthermic ranges. Benefits to HIFU include heating specificity (MRI guided HIFU) and the ability to heat deep-seated tumors. Some of the current heating devices are used to heat more superficial tumors, so tissue specificity is lost when attempting to heat tumors deeper within the tissue. Disadvantages of HIFU include difficulty in maintaining a constant temperature in larger lesions and limitations to the types of lesions that can be targeted. Since ultrasound is unable to propagate through air filled viscera (e.g. lung and bowel) and structures such as bone can absorb or reflect an ultrasound beam, certain lesion locations are not appropriate for HIFU treatment [213].

Specific heating to the tumor tissue can be challenging due to the location/depth of the tumor and the perfusion of the tumor, which can effectively carry heat away from the treatment site. In the case of temperature-sensitive liposomes, tissue specific heating or regional heating is necessary to avoid systemic drug release and normal tissue toxicity. Improved heating systems are in development to ensure that optimal temperatures are reached in the region of interest. Magnetoliposomes may be a solution to overcome heating specificity. These liposomes have iron oxide magnets in the lipid bilayer, and when exposed to a magnetic field the liposomal temperature exceeds transition temperature and the contents are released [214]. Using a magnetic field localized to the site of the tumor may improve on current heating techniques.

\subsection{Important Considerations for LTSL-Drug - Device Combinations}

LTSL-drug release requires elevating the temperature of the tumor. This is achieved using heating devices that deposit power into tumors using microwaves, RF or ultrasound, as described above. To achieve optimal drug performance, it is absolutely necessary to use hyperthermia devices to achieve a temperature distribution that maximizes drug delivery throughout the tumor volume. In an ideal setting, this would mean achieving uniform temperatures of $41.3^{\circ} \mathrm{C}$, which is the transition temperature of the LTSL (Fig. 7). However, this is not possible with any current hyperthermia devices, because the temperature distribution that is achieved is a complex product of the amount of power delivered by the device and perfusion, which carries heat away. There are considerable intratumoral and intertumoral heterogeneities in power deposition and perfusion, leading to non-uniform heating in all tumors. In Table $\mathbf{3}$, we have summarized thermal data that were acquired in selected prior trials that combined hyperthermia with radiotherapy. These trials had very different thermal goals than what is required for LTSL drugs. Nevertheless, they clearly show that the temperature ranges achieved in most tumor sites are within the range needed for drug release from LTSL. As is shown in Fig. (7), drug release starts at $39^{\circ} \mathrm{C}$, reaches a maximum at the transition temperature of $41.3^{\circ} \mathrm{C}$ and then decreases substantially at temperatures greater than $42^{\circ} \mathrm{C}$. Table 3 shows that minimal temperatures are most often in the range of $39^{\circ} \mathrm{C}$, but there are instances where maximal temperatures were substantially greater than $42^{\circ} \mathrm{C}$, which would not be ideal for optimal drug delivery with LTSL. However, it is possible to achieve tem- peratures in the desired range, if the thermal goals are established a priori. Adjustment of power and other machine settings can be used, along with measurements of temperature achieved, to alter the temperature distribution. More sophisticated heating devices under development now that involve non-invasive volumetric thermometry with real-time power control will maximize the likelihood that temperature objectives can be met.

\section{CONCLUSIONS/FUTURE DIRECTIONS}

Temperature-sensitive liposomes have progressed significantly since the 1970 s, but by no means has the work in this field reached its potential. Again, only one formulation, LTSL, with only one drug, doxorubicin, has made it to clinical trials. If these liposomes prove to be as effective in humans as in preclinical settings, a push for their use in the treatment of human disease should be made. The beauty of encapsulating FDA approved drugs is the smoother transition into the clinic. There are many chemotherapeutic drugs currently approved for human use meaning that there are many types of drugs waiting to be encapsulated in temperature-sensitive liposomes. Since one drug type cannot treat all cancers, variations in the chemotherapeutic drugs contained in these liposomes are needed.

Work is currently being done to broaden the applicability of temperature-sensitive liposomes. The area of encapsulating contrast agents for improved imaging modalities has definite clinical potential. LTSLs could make additional and new impact in the delivery and release of small molecule therapeutics, peptides and oligonucleotides to specific cellular molecular targets. Although heat is the trigger for drug release, other researchers are attempting to improve on drug targeting by labeling temperature-sensitive liposomes with antibodies. For example, Puri et al. [215] labeled temperature-sensitive liposomes with HER2-specific antibodies to more specifically target HER2-positive tumors (e.g. breast cancer). Advances such as these highlight the utility and versatility of liposomal-based treatments and offer the potential for effective therapeutic modalities not only for cancer, but a wide range of diseases.

\section{CONFLICT OF INTEREST}

Dr. Dewhirst is a consultant and Chair of the Scientific Advisory Board of Celsion Corporation. Dr. Needham is the inventor of the thermally sensitive liposome that is a primary subject of this review. Drs. Dewhirst and Needham hold stock and stock options in Celsion Corporation.

\section{ACKNOWLEDGEMENTS}

The authors thank Timothy P. Wakeman, PhD, Diane R. Fels, PhD, Eui Jung Moon, and Andrew N. Fontanella for providing helpful discussion and advice during the preparation of this manuscript. This work was supported by NIH grant CA42745-23.

\section{REFERENCES}

[1] Immordino ML, Dosio F, Cattel L. Stealth liposomes: review of the basic science, rationale, and clinical applications, existing and potential. Int J Nanomedicine 2006; 1: 297-315.

[2] Allen TM, Cullis PR. Drug delivery systems: entering the mainstream. Science 2004; 303: 1818-22.

[3] Torchilin VP. Recent advances with liposomes as pharmaceutical carriers. Nat Rev Drug Discov 2005; 4: 145-60. 
[4] Jesorka A, Orwar O. Liposomes: technologies and analytical applications. Annu Rev Anal Chem (Palo Alto Calif) 2008; 1: 80132.

[5] Needham D, Ponce A. In: Amiji MM, Ed. Nanotechnology for Cancer Therapy. CRC Press. 2006; pp. 677-719.

[6] Koning GA, Eggermont AM, Lindner LH, ten Hagen TL. Hyperthermia and thermosensitive liposomes for improved delivery of chemotherapeutic drugs to solid tumors. Pharm Res 2010; 27 : 1750-4.

[7] Rahman A, Carmichael D, Harris M, Roh JK. Comparative pharmacokinetics of free doxorubicin and doxorubicin entrapped in cardiolipin liposomes. Cancer Res 1986; 46: 2295-9.

[8] Davis AJ, Tannock IF. Tumor physiology and resistance to chemotherapy: repopulation and drug penetration. Cancer Treat Res 2002; 112: 1-26.

[9] Minchinton AI, Tannock IF. Drug penetration in solid tumours. Nat Rev Cancer 2006; 6: 583-92.

[10] Papahadjopoulos D, Ed. Liposomes and their uses in biology and medicine. New York: Annals of the New York Academy of Sciences; 1978.

[11] Allen TM. Liposomal drug formulations. Rationale for development and what we can expect for the future. Drugs 1998; 56: 747-56.

[12] Drummond DC, Meyer O, Hong K, Kirpotin DB, Papahadjopoulos D. Optimizing liposomes for delivery of chemotherapeutic agents to solid tumors. Pharmacol Rev 1999; 51: 691-743.

[13] Torchilin VP. Targeted polymeric micelles for delivery of poorly soluble drugs. Cell Mol Life Sci 2004; 61: 2549-59.

[14] Wang J, Mongayt D, Torchilin VP. Polymeric micelles for delivery of poorly soluble drugs: preparation and anticancer activity in vitro of paclitaxel incorporated into mixed micelles based on poly(ethylene glycol)-lipid conjugate and positively charged lipids. J Drug Target 2005; 13: 73-80.

[15] Kabanov AV, Batrakova EV, Alakhov VY. Pluronic block copolymers as novel polymer therapeutics for drug and gene delivery. J Control Release 2002; 82: 189-212.

[16] Rangel-Yagui CO, Pessoa A, Jr., Tavares LC. Micellar solubilization of drugs. J Pharm Pharm Sci 2005; 8: 147-65.

[17] Discher BM, Won YY, Ege DS, et al. Polymersomes: tough vesicles made from diblock copolymers. Science 1999; 284: 11436.

[18] Dharap SS, Qiu B, Williams GC, Sinko P, Stein S, Minko T. Molecular targeting of drug delivery systems to ovarian cancer by BH3 and LHRH peptides. J Control Release 2003; 91: 61-73.

[19] Kopecek J, Kopeckova P, Minko T, Lu ZR, Peterson CM. Water soluble polymers in tumor targeted delivery. J Control Release 2001; 74: 147-58.

[20] Duncan R, Gac-Breton S, Keane R, et al. Polymer-drug conjugates, PDEPT and PELT: basic principles for design and transfer from the laboratory to clinic. J Control Release 2001; 74: 135-46.

[21] Vasey PA, Kaye SB, Morrison R, et al. Phase I clinical and pharmacokinetic study of PK1 [N-(2-hydroxypropyl)methacrylamide copolymer doxorubicin]: first member of a new class of chemotherapeutic agents-drug-polymer conjugates. Cancer Research Campaign Phase I/II Committee. Clin Cancer Res 1999; 5: 83-94.

[22] Patri AK, Kukowska-Latallo JF, Baker JR, Jr. Targeted drug delivery with dendrimers: comparison of the release kinetics of covalently conjugated drug and non-covalent drug inclusion complex. Adv Drug Deliv Rev 2005; 57: 2203-14.

[23] Choi Y, Baker JR, Jr. Targeting cancer cells with DNA-assembled dendrimers: a mix and match strategy for cancer. Cell Cycle 2005; 4: 669-71.

[24] Fidler IJ, Raz A, Fogler WE, Kirsh R, Bugelski P, Poste G. Design of liposomes to improve delivery of macrophage-augmenting agents to alveolar macrophages. Cancer Res 1980; 40: 4460-6.

[25] Poste G, Bucana C, Raz A, Bugelski P, Kirsh R, Fidler IJ. Analysis of the fate of systemically administered liposomes and implications for their use in drug delivery. Cancer Res 1982; 42: 1412-22.

[26] Hunt CA, Rustum YM, Mayhew E, Papahadjopoulos D. Retention of cytosine arabinoside in mouse lung following intravenous administration in liposomes of different size. Drug Metab Dispos 1979; $7:$ 124-8.

[27] Klibanov AL, Maruyama K, Torchilin VP, Huang L. Amphipathic polyethyleneglycols effectively prolong the circulation time of liposomes. FEBS Lett 1990; 268: 235-7.
[28] Needham D, Nunn RS. Elastic deformation and failure of lipid bilayer membranes containing cholesterol. Biophys J 1990; 58: 997-1009.

[29] Hwang KJ, Luk KF, Beaumier PL. Hepatic uptake and degradation of unilamellar sphingomyelin/cholesterol liposomes: a kinetic study. Proc Natl Acad Sci U S A 1980; 77: 4030-4.

[30] Szoka F, Jr., Papahadjopoulos D. Comparative properties and methods of preparation of lipid vesicles (liposomes). Annu Rev Biophys Bioeng 1980; 9: 467-508.

[31] Allen TM, Hansen CB, de Menezes DEL. Pharmacokinetics of long-circulating liposomes. Adv Drug Deliv Rev 1995; 16: 267-84.

[32] Dvorak HF, Nagy JA, Dvorak JT, Dvorak AM. Identification and characterization of the blood vessels of solid tumors that are leaky to circulating macromolecules. Am J Pathol 1988; 133: 95-109.

[33] Dvorak HF, Orenstein NS, Carvalho AC, et al. Induction of a fibrin-gel investment: an early event in line 10 hepatocarcinoma growth mediated by tumor-secreted products. J Immunol 1979; 122: 166-74.

[34] Nagy JA, Brown LF, Senger DR, et al. Pathogenesis of tumor stroma generation: a critical role for leaky blood vessels and fibrin deposition. Biochim Biophys Acta 1989; 948: 305-26.

[35] Hong RL, Huang CJ, Tseng YL, et al. Direct comparison of liposomal doxorubicin with or without polyethylene glycol coating in C-26 tumor-bearing mice: is surface coating with polyethylene glycol beneficial? Clin Cancer Res 1999; 5: 3645-52.

[36] Hobbs SK, Monsky WL, Yuan F, et al. Regulation of transport pathways in tumor vessels: role of tumor type and microenvironment. Proc Natl Acad Sci U S A 1998; 95: 4607-12.

[37] Yuan F, Leunig M, Huang SK, Berk DA, Papahadjopoulos D, Jain RK. Microvascular permeability and interstitial penetration of sterically stabilized (stealth) liposomes in a human tumor xenograft. Cancer Res 1994; 54: 3352-6.

[38] Wu NZ, Da D, Rudoll TL, Needham D, Whorton AR, Dewhirst MW. Increased microvascular permeability contributes to preferential accumulation of Stealth liposomes in tumor tissue. Cancer Res 1993; 53: 3765-70.

[39] Wu NZ, Klitzman B, Rosner G, Needham D, Dewhirst MW. Measurement of material extravasation in microvascular networks using fluorescence video-microscopy. Microvasc Res 1993; 46: 231-53.

[40] Kong G, Braun RD, Dewhirst MW. Hyperthermia enables tumorspecific nanoparticle delivery: effect of particle size. Cancer Res 2000; 60: 4440-5.

[41] Dreher MR, Liu W, Michelich CR, Dewhirst MW, Yuan F, Chilkoti A. Tumor vascular permeability, accumulation, and penetration of macromolecular drug carriers. J Natl Cancer Inst 2006; 98: 335-44.

[42] Durand RE, Olive PL. Flow cytometry studies of intracellular adriamycin in single cells in vitro. Cancer Res 1981; 41: 3489-94.

[43] Nguyen-Ngoc T, Vrignaud P, Robert J. Cellular pharmacokinetics of doxorubicin in cultured mouse sarcoma cells originating from autochthonous tumors. Oncology 1984; 41: 55-60.

[44] El-Kareh AW, Secomb TW. A mathematical model for comparison of bolus injection, continuous infusion, and liposomal delivery of doxorubicin to tumor cells. Neoplasia 2000; 2: 325-38.

[45] Ponce AM, Viglianti BL, Yu D, et al. Magnetic resonance imaging of temperature-sensitive liposome release: drug dose painting and antitumor effects. J Natl Cancer Inst 2007; 99: 53-63.

[46] Kong G, Anyarambhatla G, Petros WP, et al. Efficacy of liposomes and hyperthermia in a human tumor xenograft model: importance of triggered drug release. Cancer Res 2000; 60: 6950-7.

[47] ClinicalTrials.gov Phase $1 / 2$ study of ThermoDox with approved hyperthermia in treatment of breast cancer recurrence at the chest wall (DIGNITY). [homepage on the internet]. Bethesda, MD: US National Institutes of Health; c1993-2010 [updated 2010 May 24; cited 2010 Aug 30]. Available from: http://www.clinicaltrials.gov/ ct $2 /$ show/NCT00826085?term $=$ thermodox + rcw\&rank $=1$

[48] ClinicalTrials.gov A study of ThermoDox ${ }^{\mathrm{TM}}$ in combination with radiofrequency ablation (RFA) in primary and metastatic tumors of the liver. [homepage on the internet]. Bethesda, MD: US National Institutes of Health; c1993-2010 [updated 2009 Dec 16; cited 2010 Aug 30]. Available from: http://www.clinicaltrials.gov/ct $2 /$ show/ NCT00441376?term $=$ thermodox + RFA+liver\&rank $=1$

[49] ClinicalTrials.gov Phase 3 study of ThermoDox with radiofrequency ablation (RFA) in treatment of hepatocellular carcinoma (HCC). [homepage on the internet]. Bethesda, MD: US 
National Institutes of Health; c1993-2010 [updated 2010 Aug 10; cited 2010 Aug 30]. Available from: http://www.clinicaltrials.gov/ ct2/show/NCT00617981?term $=$ NCT00617981\&rank $=1$

[50] Storm FK. Clinical hyperthermia and chemotherapy. Radiol Clin North Am 1989; 27: 621-7.

[51] Kong G, Braun RD, Dewhirst MW. Characterization of the effect of hyperthermia on nanoparticle extravasation from tumor vasculature. Cancer Res 2001; 61: 3027-32.

[52] Matteucci ML, Anyarambhatla G, Rosner G, et al. Hyperthermia increases accumulation of technetium-99m-labeled liposomes in feline sarcomas. Clin Cancer Res 2000; 6: 3748-55

[53] Gaber MH, Wu NZ, Hong K, Huang SK, Dewhirst MW, Papahadjopoulos D. Thermosensitive liposomes: extravasation and release of contents in tumor microvascular networks. Int J Radiat Oncol Biol Phys 1996; 36: 1177-87.

[54] Yatvin MB, Weinstein JN, Dennis WH, Blumenthal R. Design of liposomes for enhanced local release of drugs by hyperthermia. Science 1978; 202: 1290-3.

[55] Yatvin MB, Muhlensiepen H, Porschen W, Weinstein JN, Feinendegen LE. Selective delivery of liposome-associated cisdichlorodiammineplatinum(II) by heat and its influence on tumor drug uptake and growth. Cancer Res 1981; 41: 1602-7.

[56] Ben-Yosef R, Kapp DS. Persistent and/or late complications of combined radiation therapy and hyperthermia. Int $\mathrm{J}$ Hyperthermia 1992; 8: 733-45.

[57] Dewhirst MW, Sim DA. The utility of thermal dose as a predictor of tumor and normal tissue responses to combined radiation and hyperthermia. Cancer Res 1984; 44: 4772s-80s.

[58] Thrall DE, Prescott DM, Samulski TV, et al. Serious toxicity associated with annular microwave array induction of whole-body hyperthermia in normal dogs. Int J Hyperthermia 1992; 8: 23-32.

[59] Overgaard J, Gonzalez D, Hulshof MC, et al. Hyperthermia as an adjuvant to radiation therapy of recurrent or metastatic malignant melanoma: a multicentre randomized trial by the European Society for Hyperthermic Oncology. 1996. Int J Hyperthermia 2009; 25: 323-34.

[60] Sherar M, Liu FF, Pintilie M, et al. Relationship between thermal dose and outcome in thermoradiotherapy treatments for superficial recurrences of breast cancer: data from a phase III trial. Int J Radiat Oncol Biol Phys 1997; 39: 371-80.

[61] Leopold KA, Dewhirst M, Samulski T, et al. Relationships among tumor temperature, treatment time, and histopathological outcome using preoperative hyperthermia with radiation in soft tissue sarcomas. Int J Radiat Oncol Biol Phys 1992; 22: 989-98.

[62] Milani V, Pazos M, Issels RD, et al. Radiochemotherapy in combination with regional hyperthermia in preirradiated patients with recurrent rectal cancer. Strahlenther Onkol 2008; 184: 163-8.

[63] Wust P, Gellermann J, Harder C, et al. Rationale for using invasive thermometry for regional hyperthermia of pelvic tumors. Int $\mathrm{J}$ Radiat Oncol Biol Phys 1998; 41: 1129-37.

[64] Goldberg SN, Gazelle GS, Mueller PR. Thermal ablation therapy for focal malignancy: a unified approach to underlying principles, techniques, and diagnostic imaging guidance. AJR Am J Roentgenol 2000; 174: 323-31.

[65] Anyarambhatla GR, Needham D. Enhancement of the phase transition permeability of DPPC liposomes by incorporation of MPPC: a new temperature-sensitive liposome for use with mild hyperthermia. J Liposome Res 1999; 9: 491-506.

[66] Needham D, Anyarambhatla G, Kong G, Dewhirst MW. A new temperature-sensitive liposome for use with mild hyperthermia: characterization and testing in a human tumor xenograft model. Cancer Res 2000; 60: 1197-201

[67] Lindner LH, Eichhorn ME, Eibl $\mathrm{H}$, et al. Novel temperaturesensitive liposomes with prolonged circulation time. Clin Cancer Res 2004; 10: 2168-78.

[68] Woo J, Chiu GN, Karlsson G, et al. Use of a passive equilibration methodology to encapsulate cisplatin into preformed thermosensitive liposomes. Int J Pharm 2008; 349: 38-46.

[69] Maruyama K, Unezaki S, Takahashi N, Iwatsuru M. Enhanced delivery of doxorubicin to tumor by long-circulating thermosensitive liposomes and local hyperthermia. Biochim Biophys Acta 1993; 1149: 209-16.

[70] Gaber MH, Hong K, Huang SK, Papahadjopoulos D. Thermosensitive sterically stabilized liposomes: formulation and in vitro studies on mechanism of doxorubicin release by bovine serum and human plasma. Pharm Res 1995; 12: 1407-16.
[71] Wu NZ, Braun RD, Gaber MH, et al. Simultaneous measurement of liposome extravasation and content release in tumors. Microcirculation 1997; 4: 83-101.

[72] Huang SK, Stauffer PR, Hong $\mathrm{K}$, et al. Liposomes and hyperthermia in mice: increased tumor uptake and therapeutic efficacy of doxorubicin in sterically stabilized liposomes. Cancer Res 1994; 54: 2186-91.

[73] Needham D, Dewhirst MW. The development and testing of a new temperature-sensitive drug delivery system for the treatment of solid tumors. Adv Drug Deliv Rev 2001; 53: 285-305.

[74] Mills JK, Needham D. Lysolipid incorporation in dipalmitoylphosphatidylcholine bilayer membranes enhances the ion permeability and drug release rates at the membrane phase transition. Biochim Biophys Acta 2005; 1716: 77-96.

[75] Yarmolenko PS, Zhao Y, Landon C, et al. Comparative effects of thermosensitive doxorubicin-containing liposomes and hyperthermia in human and murine tumours. Int J Hyperthermia 2010; 26: 485-98.

[76] Manzoor AA, Lindner LH, Park JY, et al. A new paradigm for drug delivery to tumors: the importance of triggered intravascular drug release from thermally-sensitive liposomes as a mechanism to enhance drug penetration distance. 2010; (In review).

[77] Haest CW, de Gier J, van Es GA, Verkleij AJ, van Deenen LL. Fragility of the permeability barrier of Escherichia coli. Biochim Biophys Acta 1972; 288: 43-53

[78] Marsh D, Watts A, Knowles PF. Evidence for phase boundary lipid. Permeability of Tempo-choline into dimyristoylphosphatidylcholine vesicles at the phase transition. Biochemistry 1976; 15: 3570-8.

[79] Mouritsen OG, Jorgensen K, Honger T. In: Disalvo EA, Simon SA, Eds. Permeability and stability of lipid bilayers. Boca Raton: CRC Press 1995; pp. 137-60.

[80] Mouritsen OG, Zuckermann MJ. Model of interfacial melting. Phys Rev Lett 1987; 58: 389-92

[81] Papahadjopoulos D, Jacobson K, Nir S, Isac T. Phase transitions in phospholipid vesicles. Fluorescence polarization and permeability measurements concerning the effect of temperature and cholesterol. Biochim Biophys Acta 1973; 311:330-48.

[82] Mouritsen OG, Jorgensen K. Dynamical order and disorder in lipid bilayers. Chem Phys Lipids 1994; 73: 3-25.

[83] Kenworthy AK, Hristova K, Needham D, McIntosh TJ. Range and magnitude of the steric pressure between bilayers containing phospholipids with covalently attached poly(ethylene glycol). Biophys J 1995; 68: 1921-36.

[84] Hossann M, Wiggenhorn M, Schwerdt A, et al. In vitro stability and content release properties of phosphatidylglyceroglycerol containing thermosensitive liposomes. Biochim Biophys Acta 2007; 1768: 2491-9.

[85] Nicholas AR, Scott MJ, Kennedy NI, Jones MN. Effect of grafted polyethylene glycol (PEG) on the size, encapsulation efficiency and permeability of vesicles. Biochim Biophys Acta 2000; 1463: 167-78.

[86] Wright AM. Drug loading and release from a thermally sensitive liposome. Department of Mechanical Engineering and Materials Science Doctoral Thesis. Durham: Duke University 2006.

[87] Li L, ten Hagen TL, Schipper D, et al. Triggered content release from optimized stealth thermosensitive liposomes using mild hyperthermia. J Control Release 2010; 143: 274-9.

[88] Needham D, Stoicheva N, Zhelev DV. Exchange of monooleoylphosphatidylcholine as monomer and micelle with membranes containing poly(ethylene glycol)-lipid. Biophys J 1997; 73: $2615-29$

[89] Ickenstein LM. Triggered drug release from thermosemsitive liposomes. Division of Pharmarceutics and Biopharmaceutics Doctoral Thesis. Vancouver, Canada: University of British Columbia 2003.

[90] Tristram-Nagle S, Wiener MC, Yang CP, Nagle JF. Kinetics of the subtransition in dipalmitoylphosphatidylcholine. Biochemistry 1987; 26: 4288-94

[91] Taylor KMG, Craig DQM. In: Torchilin VP, Weissig V, Eds Liposomes: a practical approach. 2nd ed. Oxford, Oxford University Press 2003; pp. 79-103.

[92] New RRC, Ed. Liposomes: a practical approach. Oxford: IRL Press; 1990.

[93] Kim DH, Costello MJ, Duncan PB, Needham D. Mechanica properties and microstructure of polycrystalline phospholipid 
monolayer shells: novel solid microparticles. Langmuir 2003; 19 : 8455-66.

[94] Ickenstein LM, Arfvidsson MC, Needham D, Mayer LD, Edwards K. Disc formation in cholesterol-free liposomes during phase transition. Biochim Biophys Acta 2003; 1614: 135-8.

[95] Mills JK. Triggered release of liposome contents: mechanism involved in membrane permeability and compromise. Department of Mechanical Engineering and Materials Science Doctoral Thesis. Durham: Duke University 2002

[96] Langner M, Hui SW. Dithionite penetration through phospholipid bilayers as a measure of defects in lipid molecular packing. Chem Phys Lipids 1993; 65: 23-30.

[97] Allen TM, Chonn A. Large unilamellar liposomes with low uptake into the reticuloendothelial system. FEBS Lett 1987; 223: 42-6.

[98] Allen TM, Hansen C, Rutledge J. Liposomes with prolonged circulation times: factors affecting uptake by reticuloendothelial and other tissues. Biochim Biophys Acta 1989; 981: 27-35.

[99] Mayer LD, Tai LC, Bally MB, Mitilenes GN, Ginsberg RS, Cullis PR. Characterization of liposomal systems containing doxorubicin entrapped in response to $\mathrm{pH}$ gradients. Biochim Biophys Acta 1990; 1025: 143-51.

[100] Dewhirst MW, Tso CY, Oliver R, Gustafson CS, Secomb TW, Gross JF. Morphologic and hemodynamic comparison of tumor and healing normal tissue microvasculature. Int J Radiat Oncol Biol Phys 1989; 17: 91-9.

[101] Chen Q, Tong S, Dewhirst MW, Yuan F. Targeting tumor microvessels using doxorubicin encapsulated in a novel thermosensitive liposome. Mol Cancer Ther 2004; 3: 1311-7.

[102] Banno B, Ickenstein LM, Chiu GN, et al. The functional roles of poly(ethylene glycol)-lipid and lysolipid in the drug retention and release from lysolipid-containing thermosensitive liposomes in vitro and in vivo. J Pharm Sci 2010; 99: 2295-308.

[103] Needham D. Temperature-sensitive liposomal formulation. US patent 6200598, March 2001

[104] Needham D. Temperature-sensitive liposomal formulation. US patent 6726925, April 2004.

[105] Celsion [homepage on the internet]. Columbia, MD: Celsion Corporation, Inc.; c2000-2010 [updated 2010 March 10; cited 2010 2010 Aug 8]. Available from: http//:www.celsion.com

[106] Li X, Hirsh DJ, Cabral-Lilly D, et al. Doxorubicin physical state in solution and inside liposomes loaded via a $\mathrm{pH}$ gradient. Biochim Biophys Acta 1998; 1415: 23-40.

[107] Bally MB, Cullis PR, Hope MJ, Madden TD, Mayer LD. Encapsulation of antineoplastic agents in liposomes. US patent 5077056, Dec 1991.

[108] Barenholz Y, Amselem S. Liposome Technology. 2nd ed. Boca Raton: CRC Press 1993

[109] Barenolz Y, Haran G. Method of amphiphatic drug loading in liposomes by pH gradient. US patent 5192549, March 1993.

[110] Evans E, Kwok R. Mechanical calorimetry of large dimyristoylphosphatidylcholine vesicles in the phase transition region. Biochemistry 1982; 21: 4874-9.

[111] Needham D, Evans E. Structure and mechanical properties of giant lipid (DMPC) vesicle bilayers from 20 degrees $C$ below to 10 degrees $\mathrm{C}$ above the liquid crystal-crystalline phase transition at 24 degrees C. Biochemistry 1988; 27: 8261-9.

[112] Rand RP, Pangborn WA, Purdon AD, Tinker DO. Lysolecithin and cholesterol interact stoichiometrically forming bimolecular lamellar structures in the presence of excess water, of lysolecithin or cholesterol. Can J Biochem 1975; 53: 189-95.

[113] Klopfenstein WE, de Kruyff B, Verkleij AJ, Demel RA, van Deenen LLM. Differential scanning calorimetry on mixtures of lecithin, lysolecithin and cholesterol. Chem Phys Lipids 1974; 13: 215-22.

[114] Ramsammy LS, Brockerhoff H. Lysophosphatidylcholinecholesterol complex. J Biol Chem 1982; 257: 3570-4.

[115] Ramsammy LS, Volwerk H, Lipton LC, Brockerhoff $H$. Association of cholesterol with lysophosphatidylcholine. Chem Phys Lipids 1983; 32: 83-9.

[116] Zhelev DV. Material property characteristics for lipid bilayers containing lysolipid. Biophys J 1998; 75: 321-30.

[117] Sandstrom MC, Ickenstein LM, Mayer LD, Edwards K. Effects of lipid segregation and lysolipid dissociation on drug release from thermosensitive liposomes. J Control Release 2005; 107: 131-42.
[118] Zhelev DV, Stoicheva N, Scherrer P, Needham D. Interaction of synthetic HA2 influenza fusion peptide analog with model membranes. Biophys J 2001; 81: 285-304.

[119] Zhelev DV, Needham D. Interactions of $\mathrm{pH}$-sensitive peptides and polymers with lipid bilayers: Binding and membrane stability. In: Current Topics in Membranes. Edited by S. A. Simon and T. J. McInotosh. USA: Academic Press, 2002; vol. 52: pp. 437-64.

[120] Gland JL, Somorjai GA. Low energy electron diffraction and work function studies of benzene, naphthalene and pyridine adsorbed on Pt(111) and Pt(100) single crystal surfaces. Surf Sci 1973; 38: 15786.

[121] Leszczynski J, Zerner MC. Molecular structure and vibrational spectra of dithionite ion by ab initio calculations. Chem Phys Lett 1989; 159: 143-7.

[122] Lindner LH, Hossann M, Vogeser M, et al. Dual role of hexadecylphosphocholine (miltefosine) in thermosensitive liposomes: active ingredient and mediator of drug release. J Control Release 2008; 125: 112-20.

[123] Hayashi H, Kono K, Takagishi T. Temperature-dependent associating property of liposomes modified with a thermosensitive polymer. Bioconjug Chem 1998; 9: 382-9

[124] Kono K, Yoshino K, Takagishi T. Effect of poly(ethylene glycol) grafts on temperature-sensitivity of thermosensitive polymermodified liposomes. J Control Release 2002; 80: 321-32.

[125] Paasonen L, Romberg B, Storm G, Yliperttula M, Urtti A, Hennink WE. Temperature-sensitive poly(N-(2-hydroxypropyl)methacrylamide mono/dilactate)-coated liposomes for triggered contents release. Bioconjug Chem 2007; 18: 2131-6.

[126] Presant CA, Proffitt RT, Teplitz RL, Williams LE, Tin GW. Method of delivering micellular particles encapsulating chemotherapeutic agents to tumors in a body. US patent 5441745 , Aug 1995.

[127] Presant CA, Proffitt RT, Teplitz RL, Williams LE, Tin GW. Method of targeting a specific location in a body. US patent 5435989, July 1995.

[128] Presant CA, Proffitt RT. Method of targeting tumors in humans. United States patent 5019369, May 1991.

[129] Alberts DS, Muggia FM, Carmichael J, et al. Efficacy and safety of liposomal anthracyclines in phase I/II clinical trials. Semin Oncol 2004; 31: 53-90.

[130] Mrozek E, Rhoades CA, Allen J, Hade EM, Shapiro CL. Phase I trial of liposomal encapsulated doxorubicin (Myocet; D-99) and weekly docetaxel in advanced breast cancer patients. Ann Oncol 2005; 16: 1087-93

[131] Gelmon KA, Tolcher A, Diab AR, et al. Phase I study of liposomal vincristine. J Clin Oncol 1999; 17: 697-705.

[132] Sarris AH, Hagemeister F, Romaguera J, et al. Liposomal vincristine in relapsed non-Hodgkin's lymphomas: early results of an ongoing phase II trial. Ann Oncol 2000; 11: 69-72.

[133] Ryan CW, Fleming GF, Janisch L, Ratain MJ. A phase I study of liposomal doxorubicin (Doxil) with topotecan. Am J Clin Oncol 2000; 23: 297-300.

[134] Skubitz KM. Phase II trial of pegylated-liposomal doxorubicin (Doxil) in sarcoma. Cancer Invest 2003; 21: 167-76.

[135] Martin FJ. Methods for determining liposome bioequivalence. US patent 20100209348, Aug 2010

[136] Kaasgaard T, Andresen TL, Jensen SS, Holte RO, Jensen LT, Jorgensen $\mathrm{K}$. Liposomes containing alkylated methotrexate analogues for phospholipase $\mathrm{A}(2)$ mediated tumor targeted drug delivery. Chem Phys Lipids 2009; 157: 94-103.

[137] Andresen TL, Davidsen J, Begtrup M, Mouritsen OG, Jorgensen K. Enzymatic release of antitumor ether lipids by specific phospholipase A2 activation of liposome-forming prodrugs. J Med Chem 2004; 47: 1694-703.

[138] Jorgensen K, Davidsen J, Mouritsen OG. Biophysical mechanisms of phospholipase A2 activation and their use in liposome-based drug delivery. FEBS Lett 2002; 531: 23-7.

[139] Yuan F, Salehi HA, Boucher Y, Vasthare US, Tuma RF, Jain RK. Vascular permeability and microcirculation of gliomas and mammary carcinomas transplanted in rat and mouse cranial windows. Cancer Res 1994; 54: 4564-8

[140] Kleiter MM, Yu D, Mohammadian LA, et al. A tracer dose of technetium-99m-labeled liposomes can estimate the effect of hyperthermia on intratumoral doxil extravasation. Clin Cancer Res 2006; 12: 6800-7. 
[141] Weinstein JN, Magin RL, Yatvin MB, Zaharko DS. Liposomes and local hyperthermia: selective delivery of methotrexate to heated tumors. Science 1979; 204: 188-91.

[142] Leal BZ, Meltz ML, Mohan N, Kuhn J, Prihoda TJ, Herman TS. Interaction of hyperthermia with Taxol in human MCF-7 breast adenocarcinoma cells. Int J Hyperthermia 1999; 15: 225-36.

[143] Alberts DS, Peng YM, Chen HS, Moon TE, Cetas TC, Hoeschele JD. Therapeutic synergism of hyperthermia-cis-platinum in a mouse tumor model. J Natl Cancer Inst 1980; 65: 455-61.

[144] Hahn GM. Potential for therapy of drugs and hyperthermia. Cancer Res 1979; 39: 2264-8.

[145] Chen Q, Krol A, Wright A, Needham D, Dewhirst MW, Yuan F. Tumor microvascular permeability is a key determinant for antivascular effects of doxorubicin encapsulated in a temperature sensitive liposome. Int J Hyperthermia 2008; 24: 475-82.

[146] Sharma D, Chelvi TP, Kaur J, Ralhan R. Thermosensitive liposomal taxol formulation: heat-mediated targeted drug delivery in murine melanoma. Melanoma Res 1998; 8: 240-4.

[147] Chelvi PT, Jain SK, Ralhan R. Heat-mediated selective delivery of liposome-associated melphalan in murine melanoma. Melanoma Res 1995; 5: 321-6.

[148] Chelvi TP, Jain SK, Ralhan R. Hyperthermia-mediated targeted delivery of thermosensitive liposome-encapsulated melphalan in murine tumors. Oncol Res 1995; 7: 393-8

[149] Zhu L, Huo Z, Wang L, Tong X, Xiao Y, Ni K. Targeted delivery of methotrexate to skeletal muscular tissue by thermosensitive magnetoliposomes. Int J Pharm 2009; 370: 136-43.

[150] Tiwari SB, Pai RM, Udupa N. Temperature sensitive liposomes of plumbagin: characterization and in vivo evaluation in mice bearing melanoma B16F1. J Drug Target 2002; 10: 585-91.

[151] Chelvi TP, Ralhan R. Designing of thermosensitive liposomes from natural lipids for multimodality cancer therapy. Int J Hyperthermia 1995; $11: 685-95$

[152] Hosokawa T, Sami M, Kato Y, Hayakawa E. Alteration in the temperature-dependent content release property of thermosensitive liposomes in plasma. Chem Pharm Bull (Tokyo) 2003; 51: 122732.

[153] Yuyama Y, Tsujimoto M, Fujimoto Y, Oku N. Potential usage of thermosensitive liposomes for site-specific delivery of cytokines. Cancer Lett 2000; 155: 71-7.

[154] Kong G, Dewhirst MW. Hyperthermia and liposomes. Int J Hyperthermia 1999; 15: 345-70.

[155] Palmer GM, Boruta RJ, Viglianti BL, Lan L, Spasojevic I, Dewhirst MW. Non-invasive monitoring of intra-tumor drug concentration and therapeutic response using optical spectroscopy. J Control Release 2010; 142: 457-64.

[156] Frederiksen LJ, Siemens DR, Heaton JP, Maxwell LR, Adams MA, Graham $\mathrm{CH}$. Hypoxia induced resistance to doxorubicin in prostate cancer cells is inhibited by low concentrations of glyceryl trinitrate. J Urol 2003; 170: 1003-7.

[157] Lindner LH, Reinl HM, Schlemmer M, Stahl R, Peller M. Paramagnetic thermosensitive liposomes for MR-thermometry. Int J Hyperthermia 2005; 21: 575-88.

[158] Zhaowu Z, Xiaoli W, Yangde Z, Xingyan L, Weihua Z, Nianfeng L. Preparation and characterization of Tegafur magnetic thermosensitive liposomes. Pharm Dev Technol 2009; 14: 350 - 7 .

[159] Wang T, Hossann M, Reinl HM, et al. In vitro characterization of phosphatidylglyceroglycerol-based thermosensitive liposomes with encapsulated 1H MR T1-shortening gadodiamide. Contrast Media Mol Imaging 2008; 3: 19-26.

[160] Peller M, Schwerdt A, Hossann M, et al. MR characterization of mild hyperthermia-induced gadodiamide release from thermosensitive liposomes in solid tumors. Invest Radiol 2008; 43: 877-92.

[161] Terreno E, Delli Castelli D, Cabella C, et al. Paramagnetic liposomes as innovative contrast agents for magnetic resonance (MR) molecular imaging applications. Chem Biodivers 2008; 5: 1901-12.

[162] Fossheim SL, Il'yasov KA, Hennig J, Bjornerud A. Thermosensitive paramagnetic liposomes for temperature control during MR imaging-guided hyperthermia: in vitro feasibility studies. Acad Radiol 2000; 7: 1107-15.

[163] Langereis S, Keupp J, van Velthoven JL, et al. A temperaturesensitive liposomal $1 \mathrm{H} \mathrm{CEST}$ and $19 \mathrm{~F}$ contrast agent for MR image-guided drug delivery. J Am Chem Soc 2009; 131: 1380-1.
[164] de Smet M, Langereis S, van den Bosch S, Grull H. Temperaturesensitive liposomes for doxorubicin delivery under MRI guidance. J Control Release 2010; 143: 120-7.

[165] Viglianti BL, Abraham SA, Michelich CR, et al. In vivo monitoring of tissue pharmacokinetics of liposome/drug using MRI: illustration of targeted delivery. Magn Reson Med 2004; 51: 1153 62.

[166] Viglianti BL, Ponce AM, Michelich CR, et al. Chemodosimetry of in vivo tumor liposomal drug concentration using MRI. Magn Reson Med 2006; 56: 1011-8.

[167] ClinicalTrials.gov [homepage on the internet]. Bethesda, MD: US National Institutes of Health; c1993-2010 [updated 2010 March; cited 2010 March 22]. Available from: www.ClinicalTrials.gov

[168] ClinicalTrials.gov Thalidomide + Dexamethasone vs. DOXIL (Doxorubicin $\mathrm{HCl}$ Liposome Injection) + Thalidomide + Dexamethasone in patients with newly diagnosed multiple myeloma. [homepage on the internet]. Bethesda, MD: US National Institutes of Health; c1993-2010 [updated 2010 Oct 18; cited 2010 Nov 18]. Available from: http://www.clinicaltrials.gov/ct2/ show/study/NCT00097981

[169] Orlowski RZ, Nagler A, Sonneveld P, et al. Randomized phase III study of pegylated liposomal doxorubicin plus bortezomib compared with bortezomib alone in relapsed or refractory multiple myeloma: combination therapy improves time to progression. $\mathrm{J}$ Clin Oncol 2007; 25: 3892-901.

[170] Gordon AN, Tonda M, Sun S, Rackoff W. Long-term survival advantage for women treated with pegylated liposomal doxorubicin compared with topotecan in a phase 3 randomized study of recurrent and refractory epithelial ovarian cancer. Gynecol Oncol 2004; 95: 1-8.

[171] Alberts DS, Liu PY, Wilczynski SP, et al. Randomized trial of pegylated liposomal doxorubicin (PLD) plus carboplatin versus carboplatin in platinum-sensitive (PS) patients with recurrent epithelial ovarian or peritoneal carcinoma after failure of initia platinum-based chemotherapy (Southwest Oncology Group Protocol S0200). Gynecol Oncol 2008; 108: 90-4.

[172] Northfelt DW, Dezube BJ, Thommes JA, et al. Pegylatedliposomal doxorubicin versus doxorubicin, bleomycin, and vincristine in the treatment of AIDS-related Kaposi's sarcoma: results of a randomized phase III clinical trial. J Clin Oncol 1998; 16: 2445-51.

[173] Garcia AA, Kempf RA, Rogers M, Muggia FM. A phase II study of Doxil (liposomal doxorubicin): lack of activity in poor prognosis soft tissue sarcomas. Ann Oncol 1998; 9: 1131-3.

[174] Ellerhorst JA, Bedikian A, Ring S, Buzaid AC, Eton O, Legha SS Phase II trial of doxil for patients with metastatic melanoma refractory to frontline therapy. Oncol Rep 1999; 6: 1097-9.

[175] Israel VP, Garcia AA, Roman L, et al. Phase II study of liposomal doxorubicin in advanced gynecologic cancers. Gynecol Oncol 2000; 78: 143-7.

[176] Garcia AA, Roman L, Muderspach L, et al. Phase I clinical trial of topotecan and pegylated liposomal doxorubicin. Cancer Invest 2005; $23: 665-70$.

[177] Iqbal S, Tsao-Wei DD, Quinn DI, et al. Phase I clinical trial of pegylated liposomal doxorubicin and docetaxel in patients with advanced solid tumors. Am J Clin Oncol 2010; 34: 27-31.

[178] Vujaskovic Z, Kim DW, Jones E, et al. A phase I/II study of neoadjuvant liposomal doxorubicin, paclitaxel, and hyperthermia in locally advanced breast cancer. Int J Hyperthermia 2010; 26: 51421

[179] Kouloulias VE, Dardoufas CE, Kouvaris JR, et al. Liposoma doxorubicin in conjunction with reirradiation and local hyperthermia treatment in recurrent breast cancer: a phase I/II trial. Clin Cancer Res 2002; 8: 374-82.

[180] Secord AA, Jones EL, Hahn CA, et al. Phase I/II trial of intravenous Doxil ${ }^{\circledR}$ and whole abdomen hyperthermia in patients with refractory ovarian cancer. Int J Hyperthermia 2005; 21: 333 47.

[181] Hauck ML, LaRue SM, Petros WP, et al. Phase I trial of doxorubicin-containing low temperature sensitive liposomes in spontaneous canine tumors. Clin Cancer Res 2006; 12: 4004-10.

[182] Susaneck SJ. Doxorubicin therapy in the dog. J Am Vet Med Assoc 1983; 182: 70-2.

[183] Vail DM, Kravis LD, Cooley AJ, Chun R, MacEwen EG Preclinical trial of doxorubicin entrapped in sterically stabilized 
liposomes in dogs with spontaneously arising malignant tumors. Cancer Chemother Pharmacol 1997; 39: 410-6.

[184] Poon RT, Borys N. Lyso-thermosensitive liposomal doxorubicin: a novel approach to enhance efficacy of thermal ablation of liver cancer. Expert Opin Pharmacother 2009; 10: 333-43.

[185] Thomas MB, Jaffe D, Choti MM, et al. Hepatocellular carcinoma: consensus recommendations of the national cancer institute clinical trials planning meeting. J Clin Oncol 2010; 28: 3994-4005.

[186] Hirata Y, Nishiwaki H. The choroidal circulation assessed by lasertargeted angiography. Prog Retin Eye Res 2006; 25: 129-47.

[187] Hirata Y, Nishiwaki H, Miura S, Ieki Y, Kiryu J, Honda Y. In vivo analysis of choroidal circulation by continuous laser-targeted angiography in the rat. Invest Ophthalmol Vis Sci 2003; 44: 31039.

[188] Asrani S, Zou S, D'Anna S, Goldberg MF, Zeimer R. Noninvasive visualization of blood flow in the choriocapillaris of the rat. Invest Ophthalmol Vis Sci 1996; 37: 312-7.

[189] Asrani S, Zeimer R. Feasibility of laser targeted photo-occlusion of ocular vessels. Br J Ophthalmol 1995; 79: 766-70.

[190] Asrani S, Zou S, D'Anna S, et al. Feasibility of laser-targeted photoocclusion of the choriocapillary layer in rats. Invest Ophthalmol Vis Sci 1997; 38: 2702-10.

[191] Ruel-Gariepy E, Shive M, Bichara A, et al. A thermosensitive chitosan-based hydrogel for the local delivery of paclitaxel. Eur J Pharm Biopharm 2004; 57: 53-63.

[192] Mulik R, Kulkarni V, Murthy RS. Chitosan-based thermosensitive hydrogel containing liposomes for sustained delivery of cytarabine. Drug Dev Ind Pharm 2009; 35: 49-56.

[193] Ruel-Gariepy E, Leclair G, Hildgen P, Gupta A, Leroux JC. Thermosensitive chitosan-based hydrogel containing liposomes for the delivery of hydrophilic molecules. J Control Release 2002; 82: 373-83.

[194] Kapp DS. Site and disease selection for hyperthermia clinical trials. Int J Hyperthermia 1986; 2: 139-56.

[195] Falk MH, Issels RD. Hyperthermia in oncology. Int J Hyperthermia 2001; 17: 1-18.

[196] Paulides MM, Bakker JF, Neufeld E, et al. Winner of the "New Investigator Award" at the European Society of Hyperthermia Oncology Meeting 2007. The HYPERcollar: a novel applicator for hyperthermia in the head and neck. Int J Hyperthermia 2007; 23: 567-76.

[197] Sneed PK, Stauffer PR, McDermott MW, et al. Survival benefit of hyperthermia in a prospective randomized trial of brachytherapy boost + /- hyperthermia for glioblastoma multiforme. Int $\mathbf{J}$ Radiat Oncol Biol Phys 1998; 40: 287-95.

[198] Fatehi D, van der Zee J, van der Wal E, Van Wieringen WN, Van Rhoon GC. Temperature data analysis for 22 patients with advanced cervical carcinoma treated in Rotterdam using radiotherapy, hyperthermia and chemotherapy: a reference point is needed. Int J Hyperthermia 2006; 22: 353-63.

[199] Gellermann J, Hildebrandt B, Issels R, et al. Noninvasive magnetic resonance thermography of soft tissue sarcomas during regional hyperthermia: correlation with response and direct thermometry. Cancer 2006; 107: 1373-82.

[200] Franckena M, Fatehi D, de Bruijne M, et al. Hyperthermia doseeffect relationship in 420 patients with cervical cancer treated with combined radiotherapy and hyperthermia. Eur J Cancer 2009; 45: 1969-78.

[201] Paulides MM, Bakker JF, Linthorst M, et al. The clinical feasibility of deep hyperthermia treatment in the head and neck: new challenges for positioning and temperature measurement. Phys Med Biol 2010; 55: 2465-80.

[202] Clarke M, Collins R, Darby S, et al. Effects of radiotherapy and of differences in the extent of surgery for early breast cancer on local recurrence and 15-year survival: an overview of the randomised trials. Lancet 2005; 366: 2087-106.

[203] Juang T, Neuman D, Schlorff J, Stauffer PR. Construction of a conformal water bolus vest applicator for hyperthermia treatment of superficial skin cancer. Conf Proc IEEE Eng Med Biol Soc 2004; 5: 3467-70.

[204] Boesch CE, Meyer T, Waschke L, et al. Long-term outcome of hyperthermic isolated limb perfusion (HILP) in the treatment of locoregionally metastasised malignant melanoma of the extremities. Int J Hyperthermia 2010; 26: 16-20.

[205] Issels RD, Lindner LH, Verweij J, et al. Neo-adjuvant chemotherapy alone or with regional hyperthermia for localised high-risk soft-tissue sarcoma: a randomised phase 3 multicentre study. Lancet Oncol 2010; 11: 561-70.

[206] Jones E, Alvarez Secord A, Prosnitz LR, et al. Intra-peritoneal cisplatin and whole abdomen hyperthermia for relapsed ovarian carcinoma. Int J Hyperthermia 2006; 22: 161-72.

[207] Colombo R, Da Pozzo LF, Salonia A, et al. Multicentric study comparing intravesical chemotherapy alone and with local microwave hyperthermia for prophylaxis of recurrence of superficial transitional cell carcinoma. J Clin Oncol 2003; 21: 4270-6.

[208] Moskovitz B, Meyer G, Kravtzov A, et al. Thermo-chemotherapy for intermediate or high-risk recurrent superficial bladder cancer patients. Ann Oncol 2005; 16: 585-9.

[209] Gofrit ON, Shapiro A, Pode D, et al. Combined local bladder hyperthermia and intravesical chemotherapy for the treatment of high-grade superficial bladder cancer. Urology 2004; 63: 466-71.

[210] Guthkelch AN, Carter LP, Cassady JR, et al. Treatment of malignant brain tumors with focused ultrasound hyperthermia and radiation: results of a phase I trial. J Neurooncol 1991; 10: 271-84.

[211] Kennedy JE. High-intensity focused ultrasound in the treatment of solid tumours. Nat Rev 2005; 5: 321-7.

[212] Dromi S, Frenkel V, Luk A, et al. Pulsed-high intensity focused ultrasound and low temperature-sensitive liposomes for enhanced targeted drug delivery and antitumor effect. Clin Cancer Res 2007; 13: 2722-7.

[213] Kennedy JE, Ter Haar GR, Cranston D. High intensity focused ultrasound: surgery of the future? Br J Radiol 2003; 76: 590-9.

[214] Babincova M, Cicmanec P, Altanerova V, Altaner C, Babinec P. AC-magnetic field controlled drug release from magnetoliposomes: design of a method for site-specific chemotherapy. Bioelectrochemistry 2002; 55: 17-9.

[215] Puri A, Kramer-Marek G, Campbell-Massa R, et al. HER2-specific affibody-conjugated thermosensitive liposomes (Affisomes) for improved delivery of anticancer agents. J Liposome Res 2008; 18 : 293-307.

[216] ThermoDox ${ }^{\circledR}$, lyso-thermosensitive liposomal doxorubicin: investigator's brochure for ThermoDox ${ }^{\circledR}$ in combination with focused heat for the treatment of solid tumors Columbia, MD: Celsion Corporation 2008. 